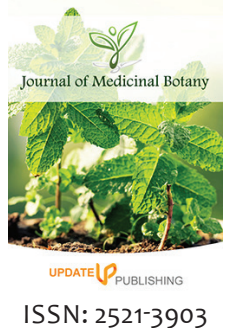

Received : August 05, 2020 Accepted : October 16, 2020 Published : October 19, 2020

* Corresponding Author: Mahamad Sayab Miya Email: sayabmiya13@gmail. com

\section{Ethnomedicinal uses of plants by major ethnic groups of Hilly Districts in Nepal: A review}

\author{
Mahamad Sayab Miya*, Sachin Timilsina, Apeksha Chhetri \\ Institute of Forestry, Pokhara Campus, Tribhuvan University, Pokhara, Nepal
}

\begin{abstract}
Plants are used as ethno-medicine by indigenous people living all around the world. In Nepal, plants are being used for healing diseases since a long period by various ethnic groups of rural areas due to difficulty in the availability of modern medicines. Many researchers have contributed to documentation of ethnomedicinal knowledge on plants in Nepal; however few studies have been carried out on hilly districts. Our study aims to review and compile all the published research documents on ethnomedicinal uses of plants by various ethnic groups of hilly districts in Nepal. Altogether 35 published documents till August 2020, accessed through Google Scholar and Research Gate were selected for our study. A total of 215 plant species from 93 families was found to be used for the treatment of 139 types of diseases by 10 ethnic groups of 13 hilly districts. Also, leaves were used for the treatment of maximum numbers of diseases (69). Plants from Fabaceae, Asteraceae, and Poaceae, etc. were used to treat major diseases like; diabetes, asthma, stomachache, fever, jaundice, etc. Traditional knowledge on medicinal uses of plants is needed to be explored and documented to preserve traditional medicinal knowledge as well as medicinal plants.
\end{abstract}

KEYWORDS: Disease, ethnomedicine, ethnic group, Indigenous people, medicinal plant

\section{INTRODUCTION}

Ethno-botany is defined as the science of interaction between people and plants [1] and deals with the study and documentation of indigenous knowledge of people on plants i.e. how plant resources are used by ethnic groups (people having their own common cultures, languages, and belief systems) [2]. Ethno-medicine deals with the study of traditional medical practices by different ethnic groups [3]. More than 20,000 species of higher plants are used for the traditional medicinal practices by indigenous people (native people) living around the world [4]. Indigenous people of developed and developing countries are using plants as a source of medicine $[5,6,7]$.

There are 1950 species of medicinal plants being used in Nepal [8], out of which 143 species are listed as commercial medicinal plants [9]. In Nepal, the lower sub-tropical region (1000-1500 m) harbors a maximum number (679 species) of medicinal plants [10]. Traditional herbal medicines have been practiced by rural people in Nepal since a long time ago because they are easily available, having no side-effects and are cheaper than modern medicines $[11,12]$. The ethnic groups have the best knowledge on the uses of plants and also transferring it to their next-generation [11]. The methods of using plants to cure several diseases vary among ethnic groups and also among healers [13]. The ethnomedicinal knowledge is being recognized worldwide because it support on innovation and formulation of many modern medicines $[11,14]$. The discovery of new drugs can be obtained through the bio-visioning of indigenous medicinal plants [15]. It is important to document and explore indigenous knowledge on medicinal plants of different ethnic groups because the knowledge might get lost with the loss of knowledgeable persons, biodiversity loss, and socio-economic changes $[16,17]$.

Many researchers have contributed on ethnomedicinal study of plants in Nepal. However, no review study has been done till now on ethnomedicinal uses of plant species by various ethnic groups of hilly districts in Nepal. Therefore, this study has attempted to review and compile all research articles on ethnomedicinal uses of plants by different ethnic groups of hilly districts in Nepal.

\section{MATERIALS AND METHODS}

The entire information was obtained from a review of several published sources including research notes and reports, academic papers, journals, and theses from 1988-2020. Google Scholar and Research gate were the primary databases for obtaining the whole data on ethnomedicinal uses of several 
tree species by ethnic groups of hilly districts in Nepal, with the keywords "Medicinal plants", "Ethnomedicinal uses", "Ethnic groups of Nepal" etc. Finally, 35 pieces of literature published until August 2020 was selected for our study.

\section{RESULTS}

There are 126 ethnic groups in Nepal, out of which 59 are officially recognized as indigenous ethnic groups [18]. Brahmin, Chhetri, Gurung, Magar, Tamang, Thami, Newar, Rai, Limbu, etc. are major indigenous ethnic groups residing on the hilly region of Nepal [18]. Documents focusing on single ethnic groups are described in our results and discussion. Ethnomedicinal uses of plants by 10 ethnic groups of 13 hilly districts are described in this paper.
The ethnic groups, their inhabited districts from where researches were carried out, numbers of plants used as a medicine, and numbers of diseases or ailments treated are mentioned in (Table 1). Plants with their scientific name, Nepali name, family, parts used, diseases treated, and used by respective ethnic groups are given in (Table 2).

\section{DISCUSSION}

Altogether 215 species of plants were found to be used for the treatment of 139 types of diseases. Whole plant or its parts i.e. rhizome, stem, bark, twig, leaf, root, fruit, bulb, seed, latex, tuber, flower, and buds of plants were used for the treatment of single or multiple diseases. The plants' parts used and numbers of diseases treated are mentioned in (Figure 1). According to our

Table 1: Ethnic groups, their inhabited districts, and numbers of (medicinal plants/diseases treated)

\begin{tabular}{|c|c|c|c|c|c|c|}
\hline S.N. & Ethnic groups & Districts (studied) & Abbreviation of districts (used on Table 2) & Numbers of plants & Numbers of diseases & Sources \\
\hline 1. & Gurung & Kaski & & 42 & 43 & [19] \\
\hline \multirow[t]{6}{*}{2.} & Magar & Parbat & $P$ & 75 & 39 & [20] \\
\hline & & Rolpa & Ro & 82 & 30 & {$[2]$} \\
\hline & & Palpa & $\mathrm{Pa}$ & 171 & 104 & [21] \\
\hline & & Gulmi & G & 161 & 123 & {$[14]$} \\
\hline & & Tanahu & $\mathrm{Ta}$ & 54 & 23 & [22] \\
\hline & & Baglung & $\mathrm{Ba}$ & 86 & 72 & [23] \\
\hline 3. & Newar & Kathmandu & & 119 & 35 & [24] \\
\hline 4. & Raji & Surkhet & & 91 & 60 & [25] \\
\hline \multirow[t]{2}{*}{5.} & Tamang & Kathmandu & K & 19 & 15 & {$[26]$} \\
\hline & & Nuwakot & N & 44 & 32 & [27] \\
\hline 6. & Thami & Illam & & 30 & 55 & [28] \\
\hline 7. & Yakkha & Dhankuta & & 30 & 47 & [29] \\
\hline 8. & Lepcha & Ilam & & 90 & 63 & [30] \\
\hline 9. & Rai & Bhojpur & & 87 & 65 & [31] \\
\hline 10. & Tangbetons & Kaski & & 60 & 64 & [32] \\
\hline
\end{tabular}

(Abbreviation for districts is used for researches carried out from more than one district.)

Table 2: Name of plants with their medicinal uses by ethnic groups of hilly districts in Nepal

\begin{tabular}{|c|c|c|c|c|c|}
\hline S.N. & $\begin{array}{l}\text { Scientific name, Nepali name and } \\
\text { Family }\end{array}$ & P.U. & Diseases treated & Ethnic groups & Sources \\
\hline 1. & $\begin{array}{l}\text { Acacia catechu (L.f.) Willd., Khayar, } \\
\text { Fabaceae }\end{array}$ & S & $\begin{array}{l}\text { Body pain and stomachache } \\
\text { Body pain }\end{array}$ & $\begin{array}{l}\text { Magar }(\mathrm{Ta}) \\
\text { Lepcha }\end{array}$ & $\begin{array}{l}{[22]} \\
{[30]}\end{array}$ \\
\hline 2. & $\begin{array}{l}\text { Acacia nilotica (L.) Delile, Babul, } \\
\text { Fabaceae }\end{array}$ & $\begin{array}{l}B, S \\
S, T \\
L\end{array}$ & $\begin{array}{l}\text { Stomachache } \\
\text { Toothache } \\
\text { Urinary tract irritation }\end{array}$ & $\begin{array}{l}\text { Raji } \\
\text { Magar }(\mathrm{Pa})\end{array}$ & $\begin{array}{l}{[25]} \\
{[33]}\end{array}$ \\
\hline 3. & Datiwan, Amaranthaceae & $\begin{array}{l}\mathrm{L} \\
\mathrm{R}\end{array}$ & $\begin{array}{l}\text { Stomachache } \\
\text { Fever, body pain, rheumatism, and pneumonia } \\
\text { Pneumonia and menstrual disorder }\end{array}$ & $\begin{array}{l}\text { Newar } \\
\text { Lepcha } \\
\text { Rai }\end{array}$ & $\begin{array}{l}{[24]} \\
{[30]} \\
{[31]}\end{array}$ \\
\hline 4. & $\begin{array}{l}\text { Aconitum forex Wall. ex Ser., Bikha, } \\
\text { Ranunculaceae }\end{array}$ & Tu & Diabetes, food poison, and stomach problem & Lepcha & {$[30]$} \\
\hline \multirow[t]{2}{*}{5.} & \multirow[t]{2}{*}{$\begin{array}{l}\text { Acorus calamus L., } \\
\text { Bojho, Acoraceae }\end{array}$} & $\mathrm{Rh}$ & $\begin{array}{l}\text { Cough and tonsillitis } \\
\text { Cough, cold, and shore throat } \\
\text { Scabies, sore throat, and appetizer }\end{array}$ & $\begin{array}{l}\text { Raji } \\
\text { Magar (Pa) } \\
\text { Yakkha }\end{array}$ & $\begin{array}{l}{[25]} \\
{[33]} \\
{[29]}\end{array}$ \\
\hline & & & $\begin{array}{l}\text { Cough } \\
\text { Cholera, diarrhoea, gastritis, cough, scabies, and toothache } \\
\text { Cough, sore throat, and scabies } \\
\text { Cough and bronchitis } \\
\text { Fever, bronchitis, stomach problems, and throat problems }\end{array}$ & $\begin{array}{l}\text { Magar }(\mathrm{Ta}) \\
\text { Lepcha } \\
\text { Magar (Ro) } \\
\text { Tamang (N) } \\
\text { Magar }(\mathrm{Ba})\end{array}$ & $\begin{array}{c}{[22]} \\
{[30,34]} \\
{[2]} \\
{[27]} \\
{[23]}\end{array}$ \\
\hline 6. & $\begin{array}{l}\text { Adhatoda vasica Nees, } \\
\text { Asuro, Acanthaceae }\end{array}$ & L & Fever & Newar & {$[24]$} \\
\hline
\end{tabular}


Table 2: (Continued)

\begin{tabular}{|c|c|c|c|c|c|}
\hline S.N. & $\begin{array}{l}\text { Scientific name, Nepali name and } \\
\text { Family }\end{array}$ & P.U. & Diseases treated & Ethnic groups & Sources \\
\hline \multirow[t]{4}{*}{7.} & \multirow[t]{4}{*}{$\begin{array}{l}\text { Aegle marmelos (L.) Correa, Bel, } \\
\text { Rutaceae }\end{array}$} & $\mathrm{F}$ & $\begin{array}{l}\text { Diarrhoea, dysentery, heat sickness, constipation, and dyspepsia } \\
\text { Diarrhoea and dysentery }\end{array}$ & $\begin{array}{l}\text { Magar (G) } \\
\text { Tamang (N) }\end{array}$ & $\begin{array}{l}{[14]} \\
{[27]}\end{array}$ \\
\hline & & & Diarrhoea & Magar (Ba) & [23] \\
\hline & & B & Stomachache & Magar $(\mathrm{Ta})$ & {$[22]$} \\
\hline & & $\mathrm{R}$ & Fever and vomiting & Magar (Ba) & [23] \\
\hline \multirow[t]{5}{*}{8.} & \multirow{5}{*}{$\begin{array}{l}\text { Ageratum conyzoides } L ., \text { Seto gandhe, } \\
\text { Asteraceae }\end{array}$} & L & Cuts and wounds & Magar (Pa) & [33] \\
\hline & & & & Magar (Ta) & {$[22]$} \\
\hline & & & & Lepcha & [30] \\
\hline & & & & Tamang (N) & [27] \\
\hline & & W & Cuts and wounds & Magar (Ba) & [23] \\
\hline \multirow[t]{2}{*}{9.} & \multirow[t]{2}{*}{ Allium cepa L., Pyaj, Amaryllidaceae } & $\mathrm{Bu}$ & Eye boils and sun stroke & Magar (Pa) & [33] \\
\hline & & & Eye boils & Magar (P) & [20] \\
\hline \multirow[t]{5}{*}{10.} & \multirow{5}{*}{$\begin{array}{l}\text { Allium sativum L., Lasun, } \\
\text { Amaryllidaceae }\end{array}$} & $\mathrm{Bu}$ & High blood pressure and altitude sickness & Gurung & [19] \\
\hline & & & Boils and gastritis & Magar $(\mathrm{Pa})$ & {$[33]$} \\
\hline & & & & Magar (P) & [20] \\
\hline & & & Flatulence and skin rashes & Tamang (N) & [27] \\
\hline & & & Cancer, gastritis, and sleep disorder & Tangbeton & [32] \\
\hline 11. & $\begin{array}{l}\text { Allium wallichii Kunth, } \\
\text { Ban lasun, Amaryllidaceae }\end{array}$ & W & Sinusitis & Tangbeton & {$[32]$} \\
\hline \multirow[t]{4}{*}{12.} & Aloe vera (L.) Burm. F., & $\mathrm{L}$ & Burned skin, gastritis, and abdominal distention & Raji & [25] \\
\hline & Ghiu kumari, Liliaceae & & Gastritis and burn & Lepcha & [30] \\
\hline & & & Burns and internal inflammation & Magar (Ba) & [23] \\
\hline & & & Burns & Tamang (N) & [27] \\
\hline \multirow[t]{3}{*}{13.} & Alstonia scholaris (L.) R. Br., Chattiwan, & B & Fever, diarrhea, dysentery, and skin diseases & Magar (Pa) & [33] \\
\hline & Apocynaceae & & Scabies, skin diseases, and tonic & Lepcha & [30] \\
\hline & & $\mathrm{S}$ & Sprain & Magar (Ta) & [22] \\
\hline \multirow[t]{2}{*}{14.} & Alternanthera sessilis (L.) R. Br. ex. DC, & $\mathrm{L}$ & Wounds & Magar (G) & [14] \\
\hline & Bhiringi jhar, Amaranthaceae & $\mathrm{R}$ & Bloody dysentery & Yakkha & [29] \\
\hline 15. & $\begin{array}{l}\text { Amaranthus viridis L., } \\
\text { Latte sag, Amaranthaceae }\end{array}$ & $\mathrm{R}$ & Leucorrhoea, pneumonia, and colic pain & Magar (Ba) & [23] \\
\hline 16. & $\begin{array}{l}\text { Amaranthus spinosus L., Kande lundo, } \\
\text { Amaranthaceae }\end{array}$ & $\mathrm{R}$ & Overheat & Magar (P) & {$[20]$} \\
\hline 17. & $\begin{array}{l}\text { Amorphophallus campanulatus (Roxb.) } \\
\text { Blume ex Decne, } \\
\text { Kaan, Araceae }\end{array}$ & $\mathrm{Bu}$ & Hydrocele, dysentery, asthma, and piles & Magar (Pa) & {$[21]$} \\
\hline \multirow[t]{3}{*}{18.} & Amomum subulatum (Roxb.) Kuntze, & Sd & Cold & Magar (P) & {$[20]$} \\
\hline & Alaichi, Zingiberaceae & & Indigestion and vomiting & Lepcha & [34] \\
\hline & & & Cough & Tangbeton & [32] \\
\hline \multirow[t]{2}{*}{19.} & Ananas comosus (L.) Merr., Bhui & $\mathrm{L}$ & Constipation & Magar (Pa) & {$[21]$} \\
\hline & & & Internal inflammation and urinary complaints & Magar (Ba) & [23] \\
\hline 20. & $\begin{array}{l}\text { Anaphalis contorta (D. Don) Hook. F., } \\
\text { Buki phul, Asteraceae }\end{array}$ & $\mathrm{R}$ & Diarrhoa & Newar & {$[24]$} \\
\hline 21. & $\begin{array}{l}\text { Annona squamosa } L_{\prime \prime} \\
\text { Sitaphal, Annonaceae }\end{array}$ & $\mathrm{F}$ & Tonic & Magar (Pa) & {$[21]$} \\
\hline \multirow[t]{5}{*}{22.} & Artemisia indica Willd., & L & Indigestion & Newar & {$[24]$} \\
\hline & Titepati, Asteraceae & & Cuts, wounds, and scabies & Magar $(\mathrm{Pa})$ & [33] \\
\hline & & & & Magar (P) & [20] \\
\hline & & & & Lepcha & [34] \\
\hline & & & Cuts, wounds, and boils & Magar (Ta) & {$[22]$} \\
\hline \multirow[t]{2}{*}{23.} & Artocarpus lakoocha Roxb., Badahar, & B & Stomachache & Magar (Ta) & [22] \\
\hline & Moraceae & Lt & Mumps & Rai & [31] \\
\hline 24. & $\begin{array}{l}\text { Andrographis paniculata (Burm. f.) } \\
\text { Wall. ex. Nees, Kaalmegh, Acanthaceae }\end{array}$ & W & Diabetes & Magar (Pa) & {$[21]$} \\
\hline 25. & $\begin{array}{l}\text { Asclepias curassavica L., Khursani } \\
\text { koshe phul, Apocynaceae }\end{array}$ & W & Antiseptic & Newar & {$[24]$} \\
\hline \multirow[t]{8}{*}{26.} & Asparagus racemosus Willd., Kurilo, & $\mathrm{Tu}$ & Diabetes & Newar & [24] \\
\hline & Asparagaceae & & Stomach problem and fracture & Raji & [35] \\
\hline & & & Increase lactation and urinary problems & Magar (Pa) & [33] \\
\hline & & & Agalactia and gastritis & Magar (P) & {$[20]$} \\
\hline & & & Laxative & Magar (Ta) & {$[22]$} \\
\hline & & & Increase lactation, tonic, and fever & Magar (Ba) & [23] \\
\hline & & & Headache, paralysis, anorexia, and lactation & Rai & [31] \\
\hline & & $S$ & Burnt area, spots on skin, and high blood pressure & Tangbeton & {$[32]$} \\
\hline
\end{tabular}


Table 2: (Continued)

\begin{tabular}{|c|c|c|c|c|c|}
\hline S.N. & $\begin{array}{l}\text { Scientific name, Nepali name and } \\
\text { Family }\end{array}$ & P.U. & Diseases treated & Ethnic groups & Sources \\
\hline \multirow[t]{3}{*}{27.} & \multirow{3}{*}{$\begin{array}{l}\text { Astilbe rivularis Buch.-Ham. ex. D.Don, } \\
\text { Budho Okhati, Saxifragaceae }\end{array}$} & \multirow[t]{3}{*}{$\mathrm{Rh}$} & Aphrodisiac and meternity problems & Newar & [24] \\
\hline & & & Back pain, body pain, and sprain & Lepcha & {$[30,34]$} \\
\hline & & & Fracture and body pain & Thami & [28] \\
\hline \multirow[t]{3}{*}{28.} & \multirow{3}{*}{$\begin{array}{l}\text { Azadirachta indica A. Juss., Neem, } \\
\text { Meliaceae }\end{array}$} & \multirow[t]{3}{*}{ L } & Toothache, malarian fever, and diarrhea & Magar (Ta) & [22] \\
\hline & & & Fever and intestinal worms & Tamang (N) & [27] \\
\hline & & & Fever & Lepcha & [34] \\
\hline 29. & Basella alba L., Poi Saag, Basellaceae & L & Constipation & Magar $(\mathrm{Pa})$ & [21] \\
\hline 30. & Bauhinia purpurea L., Tanki, Fabaceae & $\mathrm{FI}$ & Diarrhoea \& dysentery & Newar & [24] \\
\hline \multirow[t]{4}{*}{31.} & \multirow{4}{*}{$\begin{array}{l}\text { Bauhinia variegata (L.) Benth. Koiralo, } \\
\text { Fabaceae }\end{array}$} & \multirow[t]{3}{*}{ B } & \multirow[t]{2}{*}{ Diarrhoea \& dysentery } & Newar & [24] \\
\hline & & & & Raji & {$[25]$} \\
\hline & & & Dirrahoea, dysentery, piles, and liver disorders & Magar (Pa) & [33] \\
\hline & & $\mathrm{FI}$ & Dysentery and mouth sores & Yakkha & [29] \\
\hline 32. & $\begin{array}{l}\text { Begonia nepalensis (A. DC.) Warb., } \\
\text { Makar kanchi, Begoniaceae }\end{array}$ & $\mathrm{R}$ & Antihelmintic & Newar & {$[24]$} \\
\hline \multirow[t]{7}{*}{33.} & \multirow{7}{*}{$\begin{array}{l}\text { Berberis aristata DC., Chutro, } \\
\text { Berberidaceae }\end{array}$} & \multirow[t]{2}{*}{ B } & Diarrhoea & Raji & [25] \\
\hline & & & Fever and eye infection & Magar (Pa) & [33] \\
\hline & & \multirow[t]{4}{*}{$\mathrm{R}$} & Pinworm & Raji & [25] \\
\hline & & & Intestinal worms & Gurung & [19] \\
\hline & & & Wounds and inflammation & Magar (Pa) & [33] \\
\hline & & & Jaundice and diarrhea & Magar (Ro) & [2] \\
\hline & & $\mathrm{S}$ & Jaundice, fever, and skin disease & Tamang (N) & [27] \\
\hline \multirow[t]{9}{*}{34.} & \multirow{9}{*}{$\begin{array}{l}\text { Bergenia ciliata (Haw.) Sternb., } \\
\text { Pakhanbed, Saxifragaceae }\end{array}$} & \multirow[t]{5}{*}{$\mathrm{Rh}$} & Aphrodisiac, fever, maternity problem, and post pregnancy & Newar & [24] \\
\hline & & & Diarrhea and fever & Magar (Ba) & [23] \\
\hline & & & $\begin{array}{l}\text { Typhoid, dysentery, diarrhoea, vomiting, stomach ache, headache, and } \\
\text { menstrual disorder }\end{array}$ & Magar (Ro) & {$[2]$} \\
\hline & & & Cuts, wound, and stomach problems & Lepcha & [30] \\
\hline & & & Diarrhea & Tamang (K) & [26] \\
\hline & & L & Dog bite & Lepcha & [34] \\
\hline & & W & Renal calculi, fracture, and menstrual haemorrhage & Raji & [25] \\
\hline & & & Whooping cough, body pain, and sprain & Rai & [31] \\
\hline & & $S, R$ & Body pain & Magar (P) & [20] \\
\hline 35. & Betula alnoides Buch.-Ham. ex. D.Don, & B & Bone fracture, sprain, and bleeding & Magar (G) & [14] \\
\hline & Saur, Betulaceae & & Pyorrhea, tooth ache, and rabies & Lepcha & {$[30]$} \\
\hline 36. & $\begin{array}{l}\text { Blumea lacera (Burm. f.) DC., Kukure, } \\
\text { Asteraceae }\end{array}$ & L & Headache & Magar (Pa) & [21] \\
\hline 37. & $\begin{array}{l}\text { Boeravia diffusa L., Punnare Jhar, } \\
\text { Nyctaginaceae }\end{array}$ & L & Gonorrhea and bleeding & Magar (Pa) & [33] \\
\hline 38. & Bombax ceiba L., Simal, Malvaceae & B & Constipation & Raji & {$[25]$} \\
\hline & & & Dysentery and gastritis & Gurung & [19] \\
\hline & & & Aphrodisiac, diarrhea, and dysentery & Tamang (N) & [27] \\
\hline & & & Internal inflammation & Magar (Ba) & [23] \\
\hline & & $\mathrm{R}$ & Cuts and wounds & Gurung & [19] \\
\hline 39. & $\begin{array}{l}\text { Brachycorythis obcordata (Lindl. ex. } \\
\text { Wall.) Summerh., Gamdol, Orchidaceae }\end{array}$ & $\mathrm{Rh}$ & Aphrodiasic and tonic & Newar & [24] \\
\hline 40. & Brassica campestris L., Tori, & $\mathrm{Sd}$ & Back and body pain & Lepcha & [34] \\
\hline & Brassicaceae & & Cough & Rai & [31] \\
\hline 41. & $\begin{array}{l}\text { Bryophyllum pinnatum (Lam.) Oken, } \\
\text { Ajambari, Crassulaceae }\end{array}$ & L & Inflammation and cholera & Magar (Pa) & [21] \\
\hline 42. & Calotropis gigantea (L.) W.T. Aiton, & $\mathrm{F}$ & Body pain and sinusitis & Magar (G) & [14] \\
\hline & Aank, Apocynaceae & L & Boils and sprain & & \\
\hline & & & Sprain & Lepcha & {$[30]$} \\
\hline & & B & Bloody stool & Raji & [25] \\
\hline & & Lt & Sprain & & \\
\hline 43. & $\begin{array}{l}\text { Caltha palustris L., Sim Gaitihare, } \\
\text { Ranunculaceae }\end{array}$ & L & Fever & Newar & [24] \\
\hline 44. & Cannabis sativa L., Gaanja, & L & Diarrhoea and stomachache & Gurung & [19] \\
\hline & Cannabaceae & & Pain killer and stomachache & Tamang (N) & [27] \\
\hline & & & Stomach problems & Magar (Ta) & [22] \\
\hline & & $\mathrm{Sd}$ & Abdominal disorders & Newar & [24] \\
\hline & & & Blood purifier & Tangbeton & [32] \\
\hline & & & Cold & Gurung & [19] \\
\hline & & W & Abdominal pain, cuts, and wounds & & \\
\hline 45. & Capsicum annuum L., Dalle Khursani, & $\mathrm{F}$ & Gastritis & Lepcha & {$[34]$} \\
\hline & Solanaceae & & & Rai & [31] \\
\hline
\end{tabular}


Table 2: (Continued)

\begin{tabular}{|c|c|c|c|c|c|}
\hline S.N. & $\begin{array}{l}\text { Scientific name, Nepali name and } \\
\text { Family }\end{array}$ & P.U. & Diseases treated & Ethnic groups & Sources \\
\hline 46. & Carica papaya L., Mewa, Caricaceae & $\begin{array}{l}R \\
F\end{array}$ & $\begin{array}{l}\text { Renal calculus } \\
\text { Jaundice }\end{array}$ & Raji & {$[25]$} \\
\hline 47. & $\begin{array}{l}\text { Cassia fistula L., Raj brikshaya, } \\
\text { Fabaceae }\end{array}$ & $\begin{array}{l}\mathrm{F} \\
\mathrm{Sd}\end{array}$ & $\begin{array}{l}\text { Constipation } \\
\text { Rheumatism, snake bite, and stopped urine }\end{array}$ & $\begin{array}{l}\text { Raji } \\
\text { Magar (Ba) }\end{array}$ & $\begin{array}{l}{[25]} \\
{[23]}\end{array}$ \\
\hline 48. & $\begin{array}{l}\text { Cassia occidentalis (L.) Link, Thulo } \\
\text { Tapre, Fabaceae }\end{array}$ & $\begin{array}{l}\mathrm{L} \\
\mathrm{R}\end{array}$ & $\begin{array}{l}\text { Headache and skin diseases } \\
\text { Intestinal worms }\end{array}$ & Magar $(\mathrm{Pa})$ & $\begin{array}{l}{[33]} \\
{[21]}\end{array}$ \\
\hline 49. & Cassia tora L., Sano Tapre, Fabaceae & $S d$ & Insomnia & Magar (Pa) & [21] \\
\hline 50. & $\begin{array}{l}\text { Centella asiatica (L.) Urb., Ghodtapre, } \\
\text { Apiaceae }\end{array}$ & $\begin{array}{l}L \\
S\end{array}$ & $\begin{array}{l}\text { Antidote to poison, cut, wounds, and urinary trouble } \\
\text { Fever, jaundice, and stomach problem } \\
\text { Cuts, wound, snake bite, skin diseases, and severe headache } \\
\text { Skin disease } \\
\text { Fever, urinary tract infection, and body cooling } \\
\text { Fever, cough, cold, sinusitis, and pneumonia } \\
\text { Fever, jaundice, and heat sickness } \\
\text { Fever and body cooling } \\
\text { Skin disease, indigestion, and diuretic }\end{array}$ & $\begin{array}{l}\text { Newar } \\
\text { Raji } \\
\text { Gurung } \\
\text { Magar }(\mathrm{Pa}) \\
\text { Magar }(\mathrm{Ta}) \\
\text { Lepcha } \\
\text { Magar }(\mathrm{Ba}) \\
\text { Magar }(\mathrm{Pa}) \\
\text { Tamang }(\mathrm{N})\end{array}$ & $\begin{array}{l}{[24]} \\
{[25,35]} \\
{[19]} \\
{[33]} \\
{[22]} \\
{[30]} \\
{[23]} \\
{[33]} \\
{[27]}\end{array}$ \\
\hline 51. & $\begin{array}{l}\text { Cheilanthes dalhousiae (Hook.), } \\
\text { Ranisinka, Cheilanthoidae }\end{array}$ & $\begin{array}{l}\text { W } \\
\text { L }\end{array}$ & $\begin{array}{l}\text { Fever, dysentery, and cuts } \\
\text { Stomachache } \\
\text { Snake bite } \\
\text { Antiseptic }\end{array}$ & $\begin{array}{l}\text { Gurung } \\
\text { Magar (Ta) } \\
\text { Rai } \\
\text { Raji }\end{array}$ & $\begin{array}{l}{[19]} \\
{[22]} \\
{[31]} \\
{[25]}\end{array}$ \\
\hline 52. & $\begin{array}{l}\text { Chenopodium album L., Bethe, } \\
\text { Chenopodiaceae }\end{array}$ & $\begin{array}{l}\text { Sd } \\
\text { R } \\
W\end{array}$ & $\begin{array}{l}\text { Abdominal pain } \\
\text { Retention of placenta } \\
\text { Labour pain } \\
\text { Constipation }\end{array}$ & $\begin{array}{l}\text { Gurung } \\
\text { Raji } \\
\text { Lepcha }\end{array}$ & $\begin{array}{l}{[19]} \\
{[25]} \\
{[34]}\end{array}$ \\
\hline 53. & $\begin{array}{l}\text { Cinnamomum tamala (Buch.-Ham) T. } \\
\text { Nees \& Eberm., Dalchini, Lauraceae }\end{array}$ & L & $\begin{array}{l}\text { Colic pain and diarrhea } \\
\text { Digestive disorder and kidney disease } \\
\text { Stomachache and skin diseases }\end{array}$ & $\begin{array}{l}\text { Magar }(\mathrm{Pa}) \\
\text { Tangbeton } \\
\text { Magar }(\mathrm{Pa})\end{array}$ & $\begin{array}{l}{[33]} \\
{[32]} \\
{[33]}\end{array}$ \\
\hline 54. & $\begin{array}{l}\text { Cirsium verutum (D.Don) Spreng., } \\
\text { Thakal, Asteraceae }\end{array}$ & $\mathrm{R}$ & $\begin{array}{l}\text { Urinary trouble } \\
\text { Fever and marasmus }\end{array}$ & $\begin{array}{l}\text { Newar } \\
\text { Magar (Ro) }\end{array}$ & $\begin{array}{c}{[24]} \\
{[2]}\end{array}$ \\
\hline 55. & $\begin{array}{l}\text { Cissus quadrangularis L., Had jor, } \\
\text { Vitaceae }\end{array}$ & W & Cuts, wounds, and fracture & Lepcha & {$[30]$} \\
\hline 56. & $\begin{array}{l}\text { Citrus limon (L.) Osbek, Kagati, } \\
\text { Rutaceae }\end{array}$ & $\mathrm{F}$ & $\begin{array}{l}\text { Altitude sickness, vomiting, and dandruff } \\
\text { Altitude sickness }\end{array}$ & $\begin{array}{l}\text { Raji } \\
\text { Rai }\end{array}$ & $\begin{array}{l}{[25]} \\
{[31]}\end{array}$ \\
\hline 57. & $\begin{array}{l}\text { Cissampelos pareira L., Batul pate, } \\
\text { Menispermaceae }\end{array}$ & Tu & Abdominal disorders & $\begin{array}{l}\text { Raji } \\
\text { Magar (Ba) } \\
\text { Magar (Ta) }\end{array}$ & $\begin{array}{l}{[35]} \\
{[23]} \\
{[22]}\end{array}$ \\
\hline & & $\begin{array}{l}W \\
R, L\end{array}$ & $\begin{array}{l}\text { Hemorrhage } \\
\text { Gastritis, Menstrual disorder, and abdominal distention }\end{array}$ & $\begin{array}{l}\text { Newar } \\
\text { Raji }\end{array}$ & $\begin{array}{l}{[24]} \\
{[25]}\end{array}$ \\
\hline 58. & $\begin{array}{l}\text { Cleistocalyx operculatus (Roxb.) Merr. } \\
\text { \& L.M., Kyamuno, Myrtaceae }\end{array}$ & L & $\begin{array}{l}\text { Nose bleeding and sinusitis } \\
\text { Diarrhoea, dysentery, and cholera }\end{array}$ & $\begin{array}{l}\text { Rai } \\
\text { Raji } \\
\text { Lepcha }\end{array}$ & $\begin{array}{l}{[31]} \\
{[25]} \\
{[30]}\end{array}$ \\
\hline 59. & $\begin{array}{l}\text { Clematis buchannaniana DC., Pinashe } \\
\text { lahara, Ranunculaceae }\end{array}$ & $\begin{array}{l}\mathrm{L} \\
\mathrm{R}\end{array}$ & $\begin{array}{l}\text { Cough and cold } \\
\text { Sinusitis } \\
\text { Toothache }\end{array}$ & $\begin{array}{l}\text { Newar } \\
\text { Lepcha } \\
\text { Rai }\end{array}$ & $\begin{array}{l}{[24]} \\
{[34]} \\
{[31]}\end{array}$ \\
\hline 60. & $\begin{array}{l}\text { Cleome viscosa L., Ban Methi, } \\
\text { Capparaceae }\end{array}$ & $\begin{array}{l}L \\
S d\end{array}$ & $\begin{array}{l}\text { Headache and earache } \\
\text { Wounds }\end{array}$ & Magar (Pa) & [21] \\
\hline 61. & $\begin{array}{l}\text { Clerodendrum indicum (L.) Gaertn., } \\
\text { Rudilo, Verbenaceae }\end{array}$ & $\mathrm{L}$ & Cough, cold, fever, and headache & Magar (Ta) & {$[22]$} \\
\hline 62. & $\begin{array}{l}\text { Clerodendrum viscosum Vent., Bhat, } \\
\text { Lamiaceae }\end{array}$ & $\begin{array}{l}\mathrm{R} \\
\mathrm{L}\end{array}$ & $\begin{array}{l}\text { Blood dysentery } \\
\text { Wormicide }\end{array}$ & Magar (Pa) & [33] \\
\hline 63. & $\begin{array}{l}\text { Coccinia grandis (L.) Voigt., kundaru, } \\
\text { Cucurbitaceae }\end{array}$ & $\begin{array}{l}R \\
L \\
F\end{array}$ & $\begin{array}{l}\text { Labour pain } \\
\text { Liver disorders } \\
\text { Toothache }\end{array}$ & $\begin{array}{l}\text { Rai } \\
\text { Magar }(\mathrm{Pa})\end{array}$ & $\begin{array}{l}{[31]} \\
{[33]}\end{array}$ \\
\hline 64. & $\begin{array}{l}\text { Colebrookea oppositifolia Sm., Dhursuli, } \\
\text { Lamiaceae }\end{array}$ & $\mathrm{R}$ & $\begin{array}{l}\text { Conjunctivities, typhoid, and wounds } \\
\text { Epilepsy } \\
\text { Sinusitis } \\
\text { Opaqueness in cornea }\end{array}$ & $\begin{array}{l}\text { Gurung } \\
\text { Magar (Ba) } \\
\text { Raji } \\
\text { Lepcha }\end{array}$ & $\begin{array}{l}{[19]} \\
{[23]} \\
{[25]} \\
{[30]}\end{array}$ \\
\hline & & & $\begin{array}{l}\text { Nose bleeding } \\
\text { Sinuse and wounds }\end{array}$ & $\begin{array}{l}\text { Rai } \\
\text { Magar (Ba) }\end{array}$ & $\begin{array}{l}{[31]} \\
{[23]}\end{array}$ \\
\hline 65. & $\begin{array}{l}\text { Colocasia esculenta (L.) Schott, } \\
\text { Karkalo, Araceae }\end{array}$ & L & Wounds & Raji & {$[25]$} \\
\hline 66. & $\begin{array}{l}\text { Coriandrum sativum L. Dhaniya, } \\
\text { Apiaceae }\end{array}$ & $S d$ & Cold and cough & Tangbeton & [32] \\
\hline
\end{tabular}


Table 2: (Continued)

\begin{tabular}{|c|c|c|c|c|c|}
\hline S.N. & $\begin{array}{l}\text { Scientific name, Nepali name and } \\
\text { Family }\end{array}$ & P.U. & Diseases treated & Ethnic groups & Sources \\
\hline 67. & $\begin{array}{l}\text { Coriaria nepalensis Wall., Machhaino, } \\
\text { Coriariaceae }\end{array}$ & L & Antiseptic & Newar & [24] \\
\hline \multirow[t]{2}{*}{68.} & Costus speciosus (Koeing) Sm., Betlauri, & $\mathrm{R}$ & Cough, fever, pain, and tonic & Magar $(\mathrm{Ba})$ & [23] \\
\hline & Costaceae & $\mathrm{S}$ & Burning urination and stone & Rai & [31] \\
\hline 69. & $\begin{array}{l}\text { Crinum asiaticum L., Hade phool, } \\
\text { Amaryllidaceae }\end{array}$ & $\mathrm{Tu}$ & Cholera & Rai & {$[31]$} \\
\hline \multirow[t]{2}{*}{70.} & Cucumis sativus L., Kankro, & $\mathrm{Sd}$ & Malaria and pneumonia & Lepcha & {$[30]$} \\
\hline & Cucurbitaceae & $\mathrm{F}$ & Cold & & {$[34]$} \\
\hline 71. & $\begin{array}{l}\text { Cucurbita maxima Duchesne, Pharsi, } \\
\text { Cucurbitaceae }\end{array}$ & $\mathrm{F}$ & Dysentery & Rai & {$[31]$} \\
\hline 72. & $\begin{array}{l}\text { Curcuma angustifolia Roxb., Besar, } \\
\text { Zingiberaceae }\end{array}$ & $\mathrm{R}$ & Fractured and dislocated bones & Magar (Ro) & {$[2]$} \\
\hline \multirow[t]{5}{*}{73.} & Curcuma longa L. Besar, Zingiberaceae & $\mathrm{Rh}$ & Wounds and cough & Raji & {$[25]$} \\
\hline & & & Backache & Magar $(\mathrm{Pa})$ & [33] \\
\hline & & & Cold, cough, and cholera & Lepcha & [30] \\
\hline & & & Cough & Rai & [31] \\
\hline & & & Vomiting and indigestion & Tangbeton & [32] \\
\hline \multirow[t]{9}{*}{74.} & Cuscuta reflexa Roxb., Aakash beli, & W & Dandruff, jaundice, and removal of placenta & Raji & [35] \\
\hline & Convolvulaceae & & Jaundice & Magar $(\mathrm{Ta})$ & [22] \\
\hline & & & & Tamang (K) & [26] \\
\hline & & & & Rai & [31] \\
\hline & & & & Tamang (N) & [27] \\
\hline & & & & Magar (P) & {$[20]$} \\
\hline & & & Jaundice and fever & Magar (Ba) & [23] \\
\hline & & & Wound, jaundice, fracture, and stomach problems & Lepcha & {$[30]$} \\
\hline & & & Liver disorders & Magar $(\mathrm{Pa})$ & [33] \\
\hline \multirow[t]{2}{*}{75.} & Cynoglossum zeylanicum (Vahl) Thunb. & W & Cuts and wounds & Newar & [24] \\
\hline & ex. Lehm., Kanike kuro, Boraginaceae & $\mathrm{L}$ & & Gurung & [19] \\
\hline 76. & $\begin{array}{l}\text { Cyathula tomentosa (Roth) Moq., Aulo } \\
\text { kuro, Amaranthaceae }\end{array}$ & $\mathrm{R}$ & Fever & Newar & [24] \\
\hline \multirow[t]{2}{*}{77.} & Cynodon dactylon (L.) Pers., Dubo, & W & Haemorrhage & Newar & [24] \\
\hline & Poaceae & & $\begin{array}{l}\text { Inflammation of limbs, urinary tracts, gastric disorders, and } \\
\text { haemorrhage }\end{array}$ & Gurung & [19] \\
\hline 78. & $\begin{array}{l}\text { Cyperus rotundus L., Mothe, } \\
\text { Cyperaceae }\end{array}$ & $\mathrm{R}$ & Fever & Newar & [24] \\
\hline \multirow[t]{2}{*}{79.} & Dactylorhiza hatagirea (D.Don) Soo, & $\mathrm{Rh}$ & Headache, stomachache, and typhoid & Magar (Ro) & {$[2]$} \\
\hline & Panchaunle, Orchidaceaae & $\mathrm{R}$ & Piles & Tangbeton & [32] \\
\hline 80. & $\begin{array}{l}\text { Daphne papyracea Wall. ex. G.Don, } \\
\text { Lokta, Thymelaeaceae }\end{array}$ & $\mathrm{R}$ & Fever and antidode to poison & Newar & [24] \\
\hline 81. & $\begin{array}{l}\text { Datura stramonium L., Dhaturo, } \\
\text { Solanaceae }\end{array}$ & $\mathrm{L}, \mathrm{Sd}$ & Toothache, asthma, bronchitis, and insomnia & Magar (G) & [14] \\
\hline 82. & $\begin{array}{l}\text { Delphinium cooperi (Hook.f.) Jesssop, } \\
\text { Nirmasi, Ranunculaceae }\end{array}$ & $\mathrm{Rh}$ & Fever & Newar & [24] \\
\hline 83. & $\begin{array}{l}\text { Dendrocalamus hamiltonii Gambie, } \\
\text { Choya Bans, Poeceae }\end{array}$ & $\mathrm{Bd}$ & Pneumonia & Lepcha & [34] \\
\hline 84. & $\begin{array}{l}\text { Dichroa febrifuga Lour, Bhasak, } \\
\text { Hydrageaceae }\end{array}$ & $\mathrm{R}$ & Fever and antidode to poison & Newar & [24] \\
\hline \multirow[t]{4}{*}{85.} & Dioscorea bulbifera L., Githa, & $\mathrm{Tu}$ & Sore throat & Newar & [24] \\
\hline & Dioscoreaceae & & Heat sickness & Raji & {$[25]$} \\
\hline & & & Intestinal worms & Magar (Ba) & [23] \\
\hline & & $S d$ & Piles, ulcer, and dysentery & Tamang (N) & {$[27]$} \\
\hline 86. & $\begin{array}{l}\text { Dioscorea deltoidea Wall. ex Griseb., } \\
\text { Bhyakur, Dioscoreaceae }\end{array}$ & $\mathrm{Tu}$ & Antihelmintic & Newar & [24] \\
\hline \multirow[t]{5}{*}{87.} & Drymaria cordata (L.) Willd. ex Schult., & W & Cuts, wounds, and eye problem & Newar & [24] \\
\hline & Abhijalo, Caryophyllaceae & & Cold & Yakkha & [29] \\
\hline & & & Pneumonia, throat pain, sinusitis, and rashes in mouth & Thami & [28] \\
\hline & & L & $\begin{array}{l}\text { Sore throat, cough, cold, sinusitis, fever, pneumonia, cut, wounds, and } \\
\text { diarrhea }\end{array}$ & Lepcha & {$[30,34]$} \\
\hline & & & Wounds and itches & Magar (Ro) & {$[2]$} \\
\hline \multirow[t]{5}{*}{88.} & Drymeria cordta subsp. Diandra (Sw.) & W & Sinusitis and abdominal distention & Raji & {$[25]$} \\
\hline & J.A. Duke., Abhijalo, Caryophyllaceae & & Cold, cough, and stomach pain & Gurung & [19] \\
\hline & & & Sinuses and stomach disorder & Magar (Ba) & [23] \\
\hline & & & Sinusitis and headache & Rai & {$[31]$} \\
\hline & & $\mathrm{L}$ & Fever & Magar $(\mathrm{Ta})$ & [22] \\
\hline
\end{tabular}


Table 2: (Continued)

\begin{tabular}{|c|c|c|c|c|c|}
\hline S.N. & $\begin{array}{l}\text { Scientific name, Nepali name and } \\
\text { Family }\end{array}$ & P.U. & Diseases treated & Ethnic groups & Sources \\
\hline 89. & $\begin{array}{l}\text { Dryoathyrium boryanum (Willd.) Ching, } \\
\text { Kali neuro, Pteridaceae }\end{array}$ & $\mathrm{Bd}$ & Headache, fever, and stomach disorders & Magar (Ba) & {$[23]$} \\
\hline 90. & $\begin{array}{l}\text { Eclipta prostrata (L.) L., Jire jhaar, } \\
\text { Asteraceae }\end{array}$ & W & Antihelmintic & Newar & {$[24]$} \\
\hline \multirow[t]{2}{*}{91.} & \multirow{2}{*}{$\begin{array}{l}\text { Elephantopus scaber L., Sahasrabuti, } \\
\text { Asteraceae }\end{array}$} & $\mathrm{R}$ & Body cooling & Magar (Ta) & [22] \\
\hline & & $\mathrm{FI}$ & Heart disease & Rai & [31] \\
\hline 92. & $\begin{array}{l}\text { Elsholtzia flava Benth., Ban Silam, } \\
\text { Lamiaceae }\end{array}$ & $\mathrm{L}$ & Insect bite & Newar & [24] \\
\hline 93. & $\begin{array}{l}\text { Ensete glaucum (Roxb.) Cheesman, Ban } \\
\text { kera, Musaceae }\end{array}$ & $\mathrm{R}$ & Urinary tract infection & Magar (Ta) & [22] \\
\hline \multirow[t]{3}{*}{94.} & \multirow{3}{*}{$\begin{array}{l}\text { Equisetum debile Roxb. ex Vaucher, } \\
\text { Sime Jhar, Equisetaceae }\end{array}$} & W & Heat balance in body & Magar (G) & [14] \\
\hline & & & Liver disorders and constipation & Magar (Pa) & [33] \\
\hline & & $\mathrm{R}$ & Dislocation of bones & & \\
\hline 95. & $\begin{array}{l}\text { Erythrina stricta Roxb., Phaledo, } \\
\text { Fabaceae }\end{array}$ & B & Body cooling & Magar (Ta) & [22] \\
\hline \multirow[t]{3}{*}{96.} & \multirow{3}{*}{$\begin{array}{l}\text { Eupatorium adenophorum Spreng., } \\
\text { Banmara, Asteraceae }\end{array}$} & \multirow[t]{3}{*}{ L } & \multirow[t]{3}{*}{ Cuts and wounds } & Newar & [24] \\
\hline & & & & Magar (P) & {$[20]$} \\
\hline & & & & Magar (Ta) & [22] \\
\hline 97. & $\begin{array}{l}\text { Euphorbia heterophylla L., Wild } \\
\text { Poinsettia Euphorbiaceae }\end{array}$ & S & Cuts and wounds & Newar & [24] \\
\hline \multirow[t]{4}{*}{98.} & \multirow{4}{*}{$\begin{array}{l}\text { Euphorbia hirta L., Dudhe jhaar, } \\
\text { Euphorbiaceae }\end{array}$} & Lt & Antiseptic & Newar & [24] \\
\hline & & & Cuts and skin burn & Magar (Pa) & [33] \\
\hline & & L & Cuts & Magar (Ta) & [22] \\
\hline & & $\mathrm{R}$ & Cuts and wounds & Magar (P) & {$[20]$} \\
\hline \multirow[t]{2}{*}{99.} & \multirow{2}{*}{$\begin{array}{l}\text { Euphorbia royleana Boiss., Siudi, } \\
\text { Euphorbiaceae }\end{array}$} & W & Earache, boils, sprain, and muscular swellings & Gurung & [19] \\
\hline & & Lt & Stomach disorders & Magar (Ba) & [23] \\
\hline \multirow[t]{5}{*}{100.} & \multirow[t]{5}{*}{ Ficus benghalensis L. Bar, Moraceae } & $\mathrm{L}$ & Dysentery and cholera & Magar (G) & [14] \\
\hline & & B & Diabetes & & \\
\hline & & Lt & Ulcer & & \\
\hline & & & Abscess & Magar (Ba) & [23] \\
\hline & & $\mathrm{Sd}$ & Fever and cough & Tangbeton & [32] \\
\hline 101. & $\begin{array}{l}\text { Ficus lacor Buch.-Ham., Kabhro, } \\
\text { Moraceae }\end{array}$ & B & Ulcer and leucorrhoea & Magar (Ba) & [23] \\
\hline \multirow[t]{4}{*}{102.} & \multirow[t]{4}{*}{ Ficus religiosa L., Pipal, Moraceae } & \multirow[t]{2}{*}{ B } & Cuts and wounds & Magar (G) & [14] \\
\hline & & & Cough and asthma & Magar (Ba) & [23] \\
\hline & & \multirow[t]{2}{*}{$\mathrm{R}$} & Typhoid & Lepcha & {$[34]$} \\
\hline & & & Spleen sweeling & Rai & [31] \\
\hline \multirow[t]{4}{*}{103.} & \multirow{4}{*}{$\begin{array}{l}\text { Ficus semicordata Buch.-Ham. ex Sm. } \\
\text { Khanyu, Moraceae }\end{array}$} & L & Skin diseases & Magar (Pa) & [33] \\
\hline & & & Scabies & Magar (P) & [20] \\
\hline & & $\mathrm{R}$ & U rinary tract infection & Magar (Ta) & [22] \\
\hline & & Lt & Mumps & Rai & [31] \\
\hline 104. & $\begin{array}{l}\text { Fagopyrum dibotrys (D.Don.) Hara., } \\
\text { Phapar, Polygonaceae }\end{array}$ & $\mathrm{Rh}$ & Stomachache & Newar & [24] \\
\hline 105. & $\begin{array}{l}\text { Galium elegans Wall. ex Roxb., Lahare } \\
\text { Kuro, Rubiaceae }\end{array}$ & W & Antiseptic & Newar & [24] \\
\hline 106. & $\begin{array}{l}\text { Gaultheria fragrantissima Wall., } \\
\text { Dhasingre, Ericaceae }\end{array}$ & W & Rheumatism & Newar & [24] \\
\hline 107. & Girardinia diversifolia (Link) Friis, & $\mathrm{R}$ & Constipation & Magar (P) & {$[20]$} \\
\hline & Chalne sisno, Urticaceae & & Sprain & Magar (Ro) & {$[2]$} \\
\hline & & L & Diabetes & Newar & [24] \\
\hline 108. & $\begin{array}{l}\text { Hedychium spicatum Sm., Pani sarro, } \\
\text { Zingiberaceae }\end{array}$ & $\mathrm{Rh}$ & Cough and cold & Newar & [24] \\
\hline 109. & Heliotropium indicum L., Hattisude, & $\mathrm{T}$ & Rabies & Magar (Pa) & [21] \\
\hline & Boraginaceae & L & Fever, red eyeness, and conjunctivitis & & \\
\hline 110. & $\begin{array}{l}\text { Hydrocotyle javanica Thunb., Hatti } \\
\text { Paila, Apiaceae }\end{array}$ & L & Earache & Newar & {$[24]$} \\
\hline 111. & Imperata cylindrical (L.) P. Beauv., & $\mathrm{R}$ & Anthelmintic & Newar & [24] \\
\hline & Siru, Poaceae & & & Raji & {$[25,35]$} \\
\hline & & & & Lepcha & [34] \\
\hline & & & & Tamang (N) & {$[27]$} \\
\hline & & L & Diarrhoea and worms & Magar (Ro) & {$[2]$} \\
\hline 112. & Inula cappa (Buch.-Ham. ex D. Don) & $\mathrm{R}$ & Epilepsy and rheumatism & Newar & [24] \\
\hline & DC, Gaitihare, Asteraceae & & Stomachache & Magar (P) & {$[20]$} \\
\hline 113. & $\begin{array}{l}\text { Iris decora Wall., Himalayan Iris, } \\
\text { Iridaceae }\end{array}$ & $\mathrm{R}$ & Constipation & Newar & {$[24]$} \\
\hline
\end{tabular}


Table 2: (Continued)

\begin{tabular}{|c|c|c|c|c|c|}
\hline S.N. & $\begin{array}{l}\text { Scientific name, Nepali name and } \\
\text { Family }\end{array}$ & P.U. & Diseases treated & Ethnic groups & Sources \\
\hline \multirow[t]{5}{*}{114.} & \multirow{5}{*}{$\begin{array}{l}\text { Jatropha curcas L., Sajjiwan } \\
\text { Euphorbiaceae }\end{array}$} & \multirow[t]{3}{*}{$\mathrm{Lt}$} & \multirow[t]{3}{*}{ Cuts and wounds } & Magar (Ta) & [22] \\
\hline & & & & Magar (Ba) & [23] \\
\hline & & & & Yakkha & [29] \\
\hline & & W & $\begin{array}{l}\text { Syphilis, whitlow, dropsy, anasarca, pneumonia, convulsion, and } \\
\text { neuralgia }\end{array}$ & Yakkha & [29] \\
\hline & & L & Rubefacient, insecticidal, galactogogue, tumors, and scabies & & \\
\hline \multirow[t]{2}{*}{115.} & \multirow[t]{2}{*}{ Juglans regia L., Okhar, Juglandaceae } & \multirow[t]{2}{*}{ B } & Toothache & Magar (Ro) & [2] \\
\hline & & & Leprosy and skin diseases & Magar (Ba) & [23] \\
\hline 116. & $\begin{array}{l}\text { Lagenaria siceraria (Molina) Standl., } \\
\text { Lauka, Cucurbitaceae }\end{array}$ & $\mathrm{Sd}$ & Chronic cough & Magar (Ba) & {$[23]$} \\
\hline 117. & $\begin{array}{l}\text { Leucas cephalotes (Roth) Spreng., } \\
\text { Dronpuspi, Lamiaceae }\end{array}$ & $\mathrm{L}, \mathrm{FI}$ & Fever and cough & Magar (Pa) & [21] \\
\hline \multirow[t]{6}{*}{118.} & \multirow{6}{*}{$\begin{array}{l}\text { Lindera neesiana (Wall. ex Nees) Kurz, } \\
\text { Siltimur, Lauraceae }\end{array}$} & \multirow[t]{2}{*}{$\mathrm{Sd}$} & Cholera and headache & Magar (P) & [20] \\
\hline & & & Abdominal distension, altitude sickness, and gastritis & Rai & [31] \\
\hline & & \multirow[t]{3}{*}{$\mathrm{F}$} & Gastritis, cough, and cold & Thami & [28] \\
\hline & & & Cholera, gastritis, and indigestion & Lepcha & {$[30,34]$} \\
\hline & & & Fever, cough, and control poison & Magar (Ba) & [23] \\
\hline & & W & Fever and stomach disorder & Yakkha & [29] \\
\hline \multirow[t]{5}{*}{119.} & \multirow{5}{*}{$\begin{array}{l}\text { Lyonia ovalifolia (Wall.) Drude, Angeri, } \\
\text { Ericaceae }\end{array}$} & \multirow{3}{*}{$\begin{array}{l}B \\
T\end{array}$} & Scabies and skin diseases & Newar & [24] \\
\hline & & & Skin diseases & Gurung & [19] \\
\hline & & & Scabies & Magar (P) & {$[20]$} \\
\hline & & \multirow[t]{2}{*}{ L } & \multirow[t]{2}{*}{ Scabies } & Tamang (K) & [26] \\
\hline & & & & Yakkha & [29] \\
\hline 120. & $\begin{array}{l}\text { Macrotyloma uniflorum (Lam.) Verdc., } \\
\text { Gahat, Fabaceae }\end{array}$ & $\mathrm{Sd}$ & Jaundice and leucorrhoea & Magar (Pa) & {$[21]$} \\
\hline 121. & $\begin{array}{l}\text { Mahonia napaulensis DC., } \\
\text { Jamanemandro, Berberidaceae }\end{array}$ & B & Eye boils & Magar (P) & {$[20]$} \\
\hline \multirow[t]{3}{*}{122.} & \multirow{3}{*}{$\begin{array}{l}\text { Mallotus philippensis (Lam.) Muell. } \\
\text { Arg., Sindhure, Euphorbiaceae }\end{array}$} & B & Diarrhea, dysentery, and stomachache & Raji & {$[25]$} \\
\hline & & & & Magar $(\mathrm{Pa})$ & [33] \\
\hline & & $\mathrm{Sd}$ & Stomachache and body cooling & Magar $(\mathrm{Ta})$ & {$[22]$} \\
\hline 123. & Mangifera indica L., Aanp, & B & Dysentery and abdominal distension & Raji & {$[25]$} \\
\hline & Anacardiaceae & & Rheumatism and stomach ache & Magar $(\mathrm{Ta})$ & {$[22]$} \\
\hline & & & Dysentery & Rai & {$[31]$} \\
\hline & & & Gastritis & Magar (Ba) & [23] \\
\hline 124. & Melia azedarach L., Bakaino, Meliaceae & B & Anthelmintic & Newar & {$[24]$} \\
\hline & & & & Gurung & [19] \\
\hline & & $\mathrm{Sd}$ & Headache, fever, and loose bowel & Tamang (N) & [27] \\
\hline 125. & Mentha spicata L., Pudina, Lamiaceae & L & Appetizer & Gurung & [19] \\
\hline & & & Throat infection, indigestion, and appetizer & Magar $(\mathrm{Pa})$ & [33] \\
\hline & & & Vomiting, gastric disorder, appetizer, and boils & Yakkha & [29] \\
\hline & & & Cholera and stomach problems & Lepcha & {$[30]$} \\
\hline & & & Insomnia & Magar (P) & [20] \\
\hline & & W & Jaundice and heat sickness & Raji & {$[25]$} \\
\hline & & & Jaundice & Rai & {$[31]$} \\
\hline 126. & Michelia champaca L., Champ, & $\mathrm{B}$ & Wounds, scabies, and gastritis & Magar (G) & {$[14]$} \\
\hline & Magnoliaceae & $\mathrm{F}$ & Cholera, kidney problem, and fever & & \\
\hline & & $\mathrm{L}$ & Bone fracture & Magar (Ta) & [22] \\
\hline 127. & Mimosa pudica L., Lajjawati, Fabaceae & $\mathrm{R}$ & Body cooling & Magar (Ta) & [22] \\
\hline 128. & $\begin{array}{l}\text { Mirabilis jalapa L., Malatiphool, } \\
\text { Nyctaginaceae }\end{array}$ & $\mathrm{R}$ & Stomachache and gastritic & Raji & {$[25]$} \\
\hline 129. & Momordica charantia L., Tite karela, & $\mathrm{F}$ & Fever, blood purifier, and appetizer & Magar (Ba) & [23] \\
\hline & Cucurbitaceae & & High blood pressure & Tangbeton & {$[32]$} \\
\hline 130. & $\begin{array}{l}\text { Moringa oleifera Lam., Shitalchini, } \\
\text { Moringaceae }\end{array}$ & $\begin{array}{l}\mathrm{L} \\
\mathrm{Sd}\end{array}$ & $\begin{array}{l}\text { Chocked voice } \\
\text { blood pressure maintain }\end{array}$ & Magar (Pa) & {$[21]$} \\
\hline 131. & $\begin{array}{l}\text { Murraya koenigii (L.) Spreng., Mitho } \\
\text { Neem, Rutaceae }\end{array}$ & L & Eye and skin diseases & Raji & {$[25]$} \\
\hline 132. & Musa paradisiaca L., kera, Musaceae & L & Diarrhoea and dysentery & Tamang (N) & [27] \\
\hline & & S & Snake bite & Lepcha & {$[34]$} \\
\hline & & $\mathrm{Rh}$ & Internal inflammation & Magar (Ba) & [23] \\
\hline & & $\mathrm{Fl}$ & Retained placenta & Raji & {$[25]$} \\
\hline & & & & Rai & [31] \\
\hline 133. & Mussaenda macrophylla Wall., Dhobini, & $\mathrm{R}$ & Fever & Magar (Ta) & {$[22]$} \\
\hline & Rubiaceae & & Pneumonia, cough, cold, jaundice, and fever & Lepcha & {$[30]$} \\
\hline
\end{tabular}


Miya, et al.

Table 2: (Continued)

\begin{tabular}{|c|c|c|c|c|c|}
\hline S.N. & $\begin{array}{l}\text { Scientific name, Nepali name and } \\
\text { Family }\end{array}$ & P.U. & Diseases treated & Ethnic groups & Sources \\
\hline \multirow[t]{5}{*}{134.} & \multirow{5}{*}{$\begin{array}{l}\text { Myrica esculenta Buch.-Ham. ex D.Don, } \\
\text { Kafal, Myricaceae }\end{array}$} & \multirow{5}{*}{ B } & Fever & Newar & [24] \\
\hline & & & Cholera & Raji & {$[25]$} \\
\hline & & & Diarrhea, dysentery, chronic bronchitis, and rheumatic pain & Magar $(\mathrm{Pa})$ & [33] \\
\hline & & & Dysentery & Magar (P) & {$[20]$} \\
\hline & & & Bleeding from teeth and blood dysentery & Magar (Ta) & [22] \\
\hline \multirow[t]{2}{*}{135.} & \multirow{2}{*}{$\begin{array}{l}\text { Nephrolepsis cordifolia (L.) K. Presl, } \\
\text { Pani amala, Nephrolepidaceae }\end{array}$} & \multirow[t]{2}{*}{$\mathrm{Tu}$} & Indigestion, cold, cough, fever, and appetizer & Magar (Pa) & [21] \\
\hline & & & Jaundice and burning urination & Lepcha & {$[30]$} \\
\hline \multirow[t]{4}{*}{136.} & \multirow{4}{*}{$\begin{array}{l}\text { Nyctanthes arbor-tristis L. Parijat, } \\
\text { Oleaceae }\end{array}$} & \multirow[t]{2}{*}{ L } & Fever & Newar & [24] \\
\hline & & & Fever and chronic typhoid & Magar (Ba) & [23] \\
\hline & & $\mathrm{FI}$ & Diabetes and inflammation & Magar (Pa) & [21] \\
\hline & & $\mathrm{Sd}$ & Scurvy & & \\
\hline \multirow[t]{3}{*}{137.} & \multirow{3}{*}{$\begin{array}{l}\text { Ocimum gratissimum L., Bantulsi, } \\
\text { Lamiaceae }\end{array}$} & L & Gonorrhea & \multirow[t]{3}{*}{ Magar $(\mathrm{Pa})$} & \multirow[t]{3}{*}[21]{} \\
\hline & & W & Rheumatic pain and swelling & & \\
\hline & & $\mathrm{Sd}$ & Headache & & \\
\hline \multirow[t]{3}{*}{138.} & \multirow[t]{3}{*}{ Ocimum sanctum L., Tulasi, Lamiaceae } & \multirow[t]{3}{*}{ L } & Headache, sinusitis, allergies, cold, migraines, and tonsillitis & & \\
\hline & & & Cough and tonsillitis & Magar (P) & [20] \\
\hline & & & Tonic & Tangbeton & [32] \\
\hline \multirow[t]{3}{*}{139.} & \multirow{3}{*}{$\begin{array}{l}\text { Oroxylum indicum (L.) Benth. ex Kurz, } \\
\text { Tatelo, Bignoniaceae }\end{array}$} & Sd & Tyophoid & Magar (G) & [14] \\
\hline & & & Boils & Tamang (K) & {$[26]$} \\
\hline & & $\mathrm{B}$ & Jaundice & Lepcha & {$[34]$} \\
\hline 140. & $\begin{array}{l}\text { Osbeckia stellata Buch.-Ham. ex K. } \\
\text { Gawle, Angeri, Melastomataceae }\end{array}$ & L & Cuts and wounds & Newar & [24] \\
\hline 141. & Oxalis corniculata L., Chariamilo, & W & Appetizer & Newar & [24] \\
\hline & Oxalidaceae & & Body cooling & Raji & [25] \\
\hline & & & Sinusitis & Magar (P) & {$[20]$} \\
\hline & & & Fever & Magar $(\mathrm{Ta})$ & [22] \\
\hline & & & Tooth corrosion & Rai & [31] \\
\hline & & & Sinusitis, anaemia, and piles & Magar (Pa) & [33] \\
\hline & & $\mathrm{L}$ & Scurvy and Jaundice & Magar $(\mathrm{Pa})$ & [21] \\
\hline 142. & Paris Polyphylla Sm., Satuwa, & $\mathrm{Rh}$ & Cuts and wounds & Magar (Ro) & [2] \\
\hline & Melanthiaceae & & Intoxication & Magar (P) & {$[20]$} \\
\hline & & & Gastritis, ulcer, hemorrhage, wound, diarrhea, and menorrhagia & Thami & [28] \\
\hline & & & Cuts and control poison & Magar (Ba) & [23] \\
\hline & & $\mathrm{T}$ & Illness due to evil spirit & Magar (P) & {$[20]$} \\
\hline 143. & $\begin{array}{l}\text { Pedicularis gracilis Wall. ex Benth., } \\
\text { Lousewort, Orobanchaceae }\end{array}$ & $\mathrm{R}$ & Stomachache & Newar & {$[24]$} \\
\hline 144. & Phoenix humilis Royle. ex Becc., & $\mathrm{F}$ & Persistant cough & Magar (Pa) & {$[21]$} \\
\hline & Thakal, Arecaceae & $\mathrm{Tu}$ & Lung disease & & \\
\hline 145. & Phyllanthus amarus Schumach. \& & $\mathrm{L}$ & Diarrhoea & Magar (Pa) & {$[21]$} \\
\hline & Thonn., Bhuinamala, Phyllanthaceae & $\mathrm{R}$ & Fever & & \\
\hline 146. & Phyla nodiflora (L.) Greene, Jal pippali, & $\mathrm{L}$ & Gonorrhea & Magar (Pa) & {$[21]$} \\
\hline & Verbenaceae & W & Cough & & \\
\hline 147. & Phyllanthus emblica L., Amla, & $\mathrm{F}$ & Cold & Newar & [24] \\
\hline & Phyllanthaceae & & Cough and cold & Magar $(\mathrm{Ta})$ & {$[22]$} \\
\hline & & & Gastritis and stomach disorder & Lepcha & {$[30]$} \\
\hline & & & Gastritis, cough, and appetizer & Magar (Ba) & [23] \\
\hline 148. & $\begin{array}{l}\text { Picrorhiza scrophularriiflora Pennell, } \\
\text { Kutki, Plantaginaceae }\end{array}$ & $\mathrm{R}$ & Harital & Rai & [31] \\
\hline 149. & $\begin{array}{l}\text { Pimpinella diversifolia DC., Bhoke phul, } \\
\text { Apiaceae }\end{array}$ & F & Cough and cold & Newar & {$[24]$} \\
\hline 150. & Piper longum L., Pipala, Piperaceae & $\mathrm{F}$ & Cough, indigestion, and leprosy & Magar (G) & [14] \\
\hline & & & Rheumatic pain & Magar (Pa) & {$[21]$} \\
\hline & & & Cough & Magar (Ta) & [22] \\
\hline 151. & $\begin{array}{l}\text { Plantago erosa Wall., Isabgol Jhar, } \\
\text { Plantaginaceae }\end{array}$ & $\mathrm{Sd}$ & Constipation & Newar & [24] \\
\hline 152. & Plumbago zeylanica L., Chitu, & $\mathrm{R}$ & Abscess, indigestion, piles, teeth ache, and diarrhea & Magar (Ba) & [23] \\
\hline & Plumbaginaceae & & Muscle pain & Magar (Pa) & {$[21]$} \\
\hline & & W & Cough and cold & & \\
\hline 153. & Pogostemon benghalensis (Burm.f.) & $\mathrm{L}$ & Cold, cough, and fever & Magar (G) & [14] \\
\hline & Kuntz, Rudilo, Lamiaceae & $\begin{array}{l}W \\
R\end{array}$ & $\begin{array}{l}\text { Headache } \\
\text { Hematuria }\end{array}$ & Magar $(\mathrm{Pa})$ & [21] \\
\hline 154. & $\begin{array}{l}\text { Polygonatum verticillatum (L.) All., } \\
\text { Khiraula, Asparagaceae }\end{array}$ & $\mathrm{T}$ & Tonic & Newar & [24] \\
\hline
\end{tabular}


Table 2: (Continued)

\begin{tabular}{|c|c|c|c|c|c|}
\hline S.N. & $\begin{array}{l}\text { Scientific name, Nepali name and } \\
\text { Family }\end{array}$ & P.U. & Diseases treated & Ethnic groups & Sources \\
\hline \multirow[t]{4}{*}{155.} & \multirow{4}{*}{$\begin{array}{l}\text { Potentilla fulgens Wall. ex Hook., } \\
\text { Bajradanti, Rosaceae }\end{array}$} & \multirow[t]{4}{*}{$\mathrm{R}$} & \multirow[t]{4}{*}{ Toothache } & Newar & [24] \\
\hline & & & & Tamang (K) & [26] \\
\hline & & & & Magar (P) & [20] \\
\hline & & & & Tamang (N) & [27] \\
\hline \multirow[t]{2}{*}{156.} & \multirow{2}{*}{$\begin{array}{l}\text { Premna barbata Wall. ex Schauer, } \\
\text { Gineri, Lamiaceae }\end{array}$} & B & Fever & Magar $(\mathrm{Pa})$ & [21] \\
\hline & & L & Headache & Magar $(\mathrm{Ta})$ & [22] \\
\hline \multirow[t]{2}{*}{157.} & \multirow{2}{*}{$\begin{array}{l}\text { Prinsepia utilis Royle, Dhatelo, } \\
\text { Rosaceae }\end{array}$} & $\mathrm{F}$ & Body pain, joint pain, and rheumatism & Newar & [24] \\
\hline & & $\mathrm{Sd}$ & Joint ache and gout & Magar (Ro) & {$[2]$} \\
\hline \multirow[t]{3}{*}{158.} & \multirow{3}{*}{$\begin{array}{l}\text { Prunus cerasoides D.Don, Paiyu, } \\
\text { Rosaceae }\end{array}$} & \multirow[t]{3}{*}{ B } & Cut and wounds & Magar (P) & [20] \\
\hline & & & Sprains and backaches & Magar (Ro) & [2] \\
\hline & & & Body ache and stop abortion & Magar (Ba) & [23] \\
\hline \multirow[t]{9}{*}{159.} & \multirow[t]{9}{*}{ Psidium guajava L., Amba, Myrtaceae } & \multirow[t]{4}{*}{ L } & Vomiting & $\operatorname{Magar}(G)$ & [14] \\
\hline & & & Blood pressure & Magar (P) & [20] \\
\hline & & & Gastritis and flatulence & Magar $(\mathrm{Pa})$ & [33] \\
\hline & & & $\begin{array}{l}\text { Headache, wounds, ulcers, bowels, cholera, diarrhoea, indigestion, } \\
\text { rheumatism, fever, and cuts }\end{array}$ & Yakkha & [29] \\
\hline & & \multirow[t]{4}{*}{ B } & Cholera, stomach problem, and diarrhea & Lepcha & {$[30,34]$} \\
\hline & & & Diarrhoea and dysentery & Tamang (N) & [27] \\
\hline & & & & Magar (Ba) & [23] \\
\hline & & & & Rai & [31] \\
\hline & & $\mathrm{F}$ & Indigestion & Magar (G) & [14] \\
\hline \multirow[t]{2}{*}{160.} & \multirow{2}{*}{ Punica granatum L., Anar, Punicaceae } & B & Intestinal disorders & Magar (Ba) & [23] \\
\hline & & $\mathrm{F}$ & Jaundice & Tangbeton & [32] \\
\hline 161. & Rhododendron arboreum Sm., & $\mathrm{B}$ & Cuts & Gurung & [19] \\
\hline & Laligurans, Ericaceae & $\mathrm{FI}$ & Dysentery & Magar $(P)$ & {$[20]$} \\
\hline & & & Fish bone prick & Lepcha & {$[30,34]$} \\
\hline & & & & Tangbeton & [32] \\
\hline & & & & Gurung & {$[19]$} \\
\hline 162. & Rhus chinensis Mill., Bhakiamilo, & $\mathrm{F}$ & Diarrhoea & Raji & [25] \\
\hline & Anacardiaceae & & & Tamang (K) & [26] \\
\hline & & & & Rai & [31] \\
\hline 163. & Ricinus communis L., Arandi, & $\mathrm{L}$ & Body pain and antidote to snake bite & Newar & [24] \\
\hline & Euphorbiaceae & $\mathrm{Sd}$ & Rheumatism and earache & Lepcha & {$[30]$} \\
\hline 164. & $\begin{array}{l}\text { Roscoea purpurea Sm., Rashgaree, } \\
\text { Zingiberaceae }\end{array}$ & $\mathrm{Rh}$ & Aphrodisiac & Newar & {$[24]$} \\
\hline 165. & Rubia manjith Roxb., Majitho, & $\mathrm{R}$ & Antiseptic and rheumatism & Newar & [24] \\
\hline & Rubiaceae & W & Scabies & Magar (P) & [20] \\
\hline & & $\mathrm{L}$ & Cuts and wounds & Magar (Ro) & {$[2]$} \\
\hline & & & Piles & Rai & [31] \\
\hline 166. & Rubus ellipticus Sm., Ainselu, Rosaceae & $\mathrm{R}$ & Anthelmintic & Newar & [24] \\
\hline & & & Abdominal pain & Gurung & [19] \\
\hline & & & Wounds & Magar $(\mathrm{Pa})$ & [21] \\
\hline & & & U rinary tract infection & Magar (Ta) & {$[22]$} \\
\hline & & & Diarrhoea, sore throat, cholera, and gastritis & Lepcha & {$[30]$} \\
\hline & & & Fever & Magar (Ba) & [23] \\
\hline & & B & Mouth wounds and tonsillitis & Raji & [25] \\
\hline & & $\mathrm{F}$ & Appetizer & Magar (Ba) & [23] \\
\hline & & $\mathrm{Bd}$ & Cough and cold & Gurung & [19] \\
\hline & & & Fever & Magar (P) & [20] \\
\hline & & & Tongue eczema & Lepcha & [34] \\
\hline & & & Snake bite & Magar (Ba) & [23] \\
\hline 167. & Saccharum officinarum L., Ukhu, & S & Jaundice & Raji & {$[25]$} \\
\hline & Poaceae & & & Lepcha & [34] \\
\hline & & & Diarrhoea & Tangbeton & [32] \\
\hline 168. & $\begin{array}{l}\text { Saccharum spontaneoum L., Kans, } \\
\text { Poaceae }\end{array}$ & $\mathrm{R}$ & Stomachache & Magar (Ta) & {$[22]$} \\
\hline 169. & $\begin{array}{l}\text { Sarcococca coriacea (Hook.) Sweet, } \\
\text { Bhakhre Ghans, Buxaceae }\end{array}$ & L & Scabies and skin diseases & Newar & {$[24]$} \\
\hline 170. & Satyrium nepalense (Gaamdol), D.Don, & $\mathrm{R}$ & Stomachache & Magar (Ro) & [2] \\
\hline & Orchidaceae & & Fever & Newar & [24] \\
\hline 171. & $\begin{array}{l}\text { Saussurea graminifolia Wall. ex DC., } \\
\text { Saw-wort, Asteraceae }\end{array}$ & W & Kidney fever, sores, and bile disorder & Tangbeton & {$[32]$} \\
\hline 172. & $\begin{array}{l}\text { Schefflera venulosa (Wight \& Arn.) } \\
\text { Hams, Kursiulo, Araliaceae }\end{array}$ & B & Paralysis & Rai & {$[31]$} \\
\hline
\end{tabular}


Table 2: (Continued)

\begin{tabular}{|c|c|c|c|c|c|}
\hline S.N. & $\begin{array}{l}\text { Scientific name, Nepali name and } \\
\text { Family }\end{array}$ & P.U. & Diseases treated & Ethnic groups & Sources \\
\hline \multirow[t]{7}{*}{173.} & \multirow{7}{*}{$\begin{array}{l}\text { Schima wallichii (DC.) Korth., Chilaune, } \\
\text { Theaceae }\end{array}$} & \multirow{5}{*}{ B } & Anstiseptic & Newar & [24] \\
\hline & & & Fever & Tamang (N) & [27] \\
\hline & & & Piles & Rai & [31] \\
\hline & & & Fever and stomach pain & Magar (Pa) & [33] \\
\hline & & & Gastritis and healing cracks & Lepcha & {$[30]$} \\
\hline & & \multirow[t]{2}{*}{$\mathrm{F}$} & Scorpion bite & Magar (G) & [14] \\
\hline & & & Spider bite & Yakkha & [29] \\
\hline 174. & $\begin{array}{l}\text { Scindapsus officinalis Schott, Kanchiro, } \\
\text { Araceae }\end{array}$ & $\begin{array}{l}R \\
F\end{array}$ & $\begin{array}{l}\text { Cough, bronchitis, and bone fracture } \\
\text { Cuts and wounds }\end{array}$ & Magar (Pa) & [21] \\
\hline 175. & $\begin{array}{l}\text { Scoparia dulcis L., Mitha jhar, } \\
\text { Scrophulariaceae }\end{array}$ & W & Cuts, wounds, and toothache & Magar (Pa) & [21] \\
\hline 176. & $\begin{array}{l}\text { Scutellaria discolor Colebr., Ratopate, } \\
\text { Lamiaceae }\end{array}$ & $\mathrm{R}$ & Fever & Newar & [24] \\
\hline 177. & $\begin{array}{l}\text { Selinum tenuifolium Wall. ex C.B. } \\
\text { Clarke, Bhutkeshi, Apiaceae }\end{array}$ & W & Cough and cold & Newar & [24] \\
\hline \multirow[t]{2}{*}{178.} & \multirow{2}{*}{$\begin{array}{l}\text { Semecarpus anacardium L. f., Bhalayo, } \\
\text { Anacardiaceae }\end{array}$} & \multirow[t]{2}{*}{$\mathrm{F}$} & Dysentery, asthma, and acute rheumatism & Magar (G) & [14] \\
\hline & & & Wounds & Raji & [25] \\
\hline 179. & Sesamum orientale L., Til, Pedaliaceae & $\mathrm{Sd}$ & Fever & Tangbeton & [32] \\
\hline 180. & $\begin{array}{l}\text { Shorea robusta Roth, Sal, } \\
\text { Dipterocarpaceae }\end{array}$ & S & Blood dysentery & Magar (Ta) & [22] \\
\hline 181. & Sida cordifolia L., balu, Malvaceae & $\begin{array}{l}\text { W } \\
R\end{array}$ & $\begin{array}{l}\text { Rheumatic pain } \\
\text { Jaundice }\end{array}$ & Magar (Pa) & [21] \\
\hline 182. & $\begin{array}{l}\text { Smilax ovalifolia Roxb. ex D.Don, } \\
\text { Kukurdaino, Smilacaceae }\end{array}$ & $\mathrm{L}$ & Scabies, skin diseases, and meternity problems & Newar & [24] \\
\hline 183. & $\begin{array}{l}\text { Solanum aculeatissimum Jacq., } \\
\text { Kantakari, Solanceae }\end{array}$ & $\mathrm{F}$ & Jaundice & Newar & {$[24]$} \\
\hline \multirow[t]{3}{*}{184.} & \multirow[t]{3}{*}{ Solanum anguivi Lam., Bihi, Solanaceae } & \multirow[t]{3}{*}{$\mathrm{F}$} & Headache & Magar (G) & [14] \\
\hline & & & Diabetic, high blood pressure, and headache & Yakkha & [29] \\
\hline & & & Toothache, piles, and scabies & Magar (Ba) & [23] \\
\hline 185. & $\begin{array}{l}\text { Solanum indicum L., Kande bihi, } \\
\text { Solanaceae }\end{array}$ & $S d$ & Toothache & Lepcha & {$[34]$} \\
\hline \multirow[t]{3}{*}{186.} & \multirow{3}{*}{$\begin{array}{l}\text { Solanum nigrum L. Jungali bihi, } \\
\text { Solanaceae }\end{array}$} & $\mathrm{F}$ & Diabetes & Newar & [24] \\
\hline & & & Headache & Tamang (K) & [26] \\
\hline & & L & Insomnia and indigestion & Raji & [25] \\
\hline 187. & $\begin{array}{l}\text { Solidago virgaaurea L., Goldenrod, } \\
\text { Asteraceae }\end{array}$ & W & Diarrhea & Newar & [24] \\
\hline 188. & Sonchus arvensis L., Dudhi, Asteraceae & W & Diabetes & Newar & [24] \\
\hline 189. & $\begin{array}{l}\text { Spilanthes calva DC., Marethi, } \\
\text { Asteraceae }\end{array}$ & W & Toothache & Newar & {$[24]$} \\
\hline 190. & $\begin{array}{l}\text { Spiranthes sinensis (Pers.) Ames, Screw } \\
\text { Flower, Orchidaceae }\end{array}$ & $\mathrm{Rh}$ & Aphrodisiac & Newar & [24] \\
\hline \multirow[t]{4}{*}{191.} & \multirow{4}{*}{$\begin{array}{l}\text { Spondias pinnata L. f. Kurz, Amaro, } \\
\text { Anacardiaceae }\end{array}$} & B & Rheumatism & Magar (Ta) & [22] \\
\hline & & & Joint pain & Magar (Ba) & [23] \\
\hline & & $\mathrm{F}$ & Cough and rheumatism & & \\
\hline & & $\mathrm{Sd}$ & Fever & Tamang (N) & [27] \\
\hline \multirow[t]{2}{*}{192.} & Swertia angustifolia Buch.-Ham. ex & W & Anthelmintic, diarrhoea, fever, headache, and stomachache & Newar & [24] \\
\hline & D.Don, Goru tite, Gentianaceae & $\mathrm{R}$ & Antipyretic & Tamang (K) & [26] \\
\hline \multirow[t]{4}{*}{193.} & Swertia chirayita (Roxb. ex Fleming) $\mathrm{H}$. & W & Fever, malaria, cough, cold, diarrhoea, pneumonia, and diabetes & Lepcha & {$[30]$} \\
\hline & Karst, Chiraito, Gentianaceae & & Fever, typhoid, diabetes, cuts, and wounds & Magar (Ba) & [23] \\
\hline & & & Fever, pneumonia, and jaundice & Tangbeton & [32] \\
\hline & & $L, S$ & Fever & Lepcha & [34] \\
\hline 194. & Syzygium cumini (L.) Skeels, Jamun, & B & Cough, headache, and sinusitis & Magar $(\mathrm{Ta})$ & [22] \\
\hline & Myrtaceae & & Dysentery & Rai & [31] \\
\hline 195. & Tagetes erecta L., Sayapatri, Asteraceae & L & Fever & Lepcha & [34] \\
\hline 196. & Tamarindus indica L., Imali, Fabaceae & L & Inflammation, boils, and chicken pox & Magar $(\mathrm{Pa})$ & [21] \\
\hline 197. & Terminalia belerica (Gaertn.) Roxb., & $\mathrm{F}$ & Cough & Magar (Ta) & [22] \\
\hline & Barro, Combretaceae & & Measles, gastritis, tonsillitis, and stomach problems & Lepcha & [30] \\
\hline & & & Cough and throat pain & Tangbeton & [32] \\
\hline & & & Cough and bronchitis & Tamang (N) & [27] \\
\hline 198. & Terminalia chebula (Gaertn.) Retz., & $\mathrm{F}$ & Cough & Magar $(\mathrm{Ta})$ & [22] \\
\hline & Harro, Combretaceae & & Sore throat, fever, and cough & Lepcha & {$[30]$} \\
\hline & & & Cough and bronchitis & Tamang (N) & [27] \\
\hline & & & Cough, fever, and eye disease & Magar (Ba) & [23] \\
\hline & & & Gastritis and blood purifier & Tangbeton & [32] \\
\hline
\end{tabular}


Table 2: (Continued)

\begin{tabular}{|c|c|c|c|c|c|}
\hline S.N. & $\begin{array}{l}\text { Scientific name, Nepali name and } \\
\text { Family }\end{array}$ & P.U. & Diseases treated & Ethnic groups & Sources \\
\hline 199. & $\begin{array}{l}\text { Thalictrum virgatum Hook. fil. \& } \\
\text { Thomson, Daampaate, Ranunculaceae }\end{array}$ & $\mathrm{R}$ & Toothache & Newar & {$[24]$} \\
\hline \multirow[t]{3}{*}{200.} & Thespesia lampas (Cav.) Dalzell \& A. & $S, R$ & Joint and backbone pain & $\operatorname{Magar}(G)$ & [14] \\
\hline & Gibson, Ban kapas, Malvaceae & $\mathrm{R}$ & Jaundice & Tamang (N) & [27] \\
\hline & & W & Cut, wounds, sprain, and bone & Magar $(\mathrm{Ta})$ & [22] \\
\hline \multirow[t]{6}{*}{201.} & Thysanolaena maxima (Roxb.) Kuntze, & $\mathrm{R}$ & Antihelmintic & Newar & [24] \\
\hline & Amriso, Poaceae & & & Magar (Pa) & [33] \\
\hline & & & Chocking needle on foot and boils & Lepcha & [30] \\
\hline & & & Labour pain & Rai & [31] \\
\hline & & & & Raji & [25] \\
\hline & & B & Snake bite & Raji & [25] \\
\hline \multirow[t]{6}{*}{202.} & Tinospora cordifolia (Thunb.) Miers, & $\mathrm{Rh}$ & Diabetes and stomach problem & Raji & [35] \\
\hline & Gurjo, Menispermaceae & & & Newar & [24] \\
\hline & & & Stomachache & Tamang (K) & [26] \\
\hline & & & Sprain and body cooling & Magar (Ta) & [22] \\
\hline & & & Diabetes and body pain & Magar (Ba) & [23] \\
\hline & & T & Menstruation problem & Tamang (N) & [27] \\
\hline 203. & $\begin{array}{l}\text { Trichosanthes lepiniana (Naudin) Cogn., } \\
\text { Chichindo, Cucurbitaceae }\end{array}$ & $\mathrm{Sd}$ & Sore throat & Newar & {$[24]$} \\
\hline 204. & $\begin{array}{l}\text { Tridax procumbens L., Kurkure, } \\
\text { Asteraceae }\end{array}$ & W & Cuts and wounds & Magar (Pa) & [33] \\
\hline \multirow[t]{4}{*}{205.} & Trigonella foenum- graceum L., Methi, & $S d$ & Cold and cough & Raji & {$[25]$} \\
\hline & Fabaceae & & Heat sickness & Magar (Pa) & [33] \\
\hline & & & Cough & Lepcha & [34] \\
\hline & & L & Boils & Magar (Pa) & [33] \\
\hline \multirow[t]{10}{*}{206.} & Urtica dioica L., Sisnoo, Urticaceae & $\mathrm{R}$ & Labour pain and retain placenta & Raji & [25] \\
\hline & & & Fever, mental and stomach disorder & Gurung & [19] \\
\hline & & & Toothache & Magar (Pa) & [33] \\
\hline & & L & High blood pressure & Raji & [25] \\
\hline & & & Dog bite & Magar (Pa) & [21] \\
\hline & & & & Lepcha & {$[34]$} \\
\hline & & & Diabetes and rheumatism & Magar (P) & [20] \\
\hline & & $\mathrm{Bd}$ & Diabetes & Newar & [24] \\
\hline & & & Diabetes and rheumatism & Magar (Pa) & [33] \\
\hline & & W & Cuts, wounds, and fracture & Lepcha & [30] \\
\hline 207. & $\begin{array}{l}\text { Viola serpens Wall., Ghatte Ghas, } \\
\text { Violaceae }\end{array}$ & W & Cold and cough & Newar & {$[24]$} \\
\hline \multirow[t]{4}{*}{208.} & Viscum album L., Hadchur, Santalaceae & W & Cuts, wounds, and fracture & Lepcha & {$[30]$} \\
\hline & & & Fracture and body pain & Thami & [28] \\
\hline & & & Fracture & Rai & [31] \\
\hline & & L & Fracture & Magar (Ta) & [22] \\
\hline \multirow[t]{3}{*}{209.} & Vitex negundo L., Simali, Verbenaceae & L & Cold and cough & Newar & [24] \\
\hline & & & Swollen body parts & Rai & [31] \\
\hline & & $\mathrm{T}$ & Sinuses & Magar (Ba) & [23] \\
\hline \multirow[t]{7}{*}{210.} & Woodfordia fruticosa (L.) Kurtz, Dhiero, & $\mathrm{FI}$ & Cholera & Raji & {$[25]$} \\
\hline & Lythraceae & & Jaundice and dysentery & Magar $(\mathrm{Pa})$ & {$[33]$} \\
\hline & & & Dysentery & Magar $(P)$ & {$[20]$} \\
\hline & & & & Tamang (N) & [27] \\
\hline & & & & Magar (Ba) & [23] \\
\hline & & & Stomachache & Magar (Ta) & [22] \\
\hline & & & Cholera & Rai & {$[31]$} \\
\hline 211. & $\begin{array}{l}\text { Xeromphis spinosa (Thunb.) Keay, Main } \\
\text { kanda, Rubiaceae }\end{array}$ & $\mathrm{R}$ & Headache & $\operatorname{Magar}(G)$ & [14] \\
\hline 212. & $\begin{array}{l}\text { Youngia japonica (L.) DC., Dulla jhar, } \\
\text { Asteraceae }\end{array}$ & L & Indigestion & Magar (G) & {$[14]$} \\
\hline \multirow[t]{8}{*}{213.} & Zanthoxylum armatum DC., Timur, & $\mathrm{Sd}$ & Cold and gastritis & Magar (Pa) & [33] \\
\hline & Rutaceae & & Gastritis & Magar (P) & [20] \\
\hline & & & Cold, stomach disoreder, and poison & Magar (Ro) & [2] \\
\hline & & $\mathrm{F}$ & Headache, abdominal pain, appetite, and indigestion & Gurung & [19] \\
\hline & & & Cold and cough & Magar (Pa) & [21] \\
\hline & & & & Tangbeton & [32] \\
\hline & & & Joint pain & Lepcha & {$[34]$} \\
\hline & & & Fever, cough, asthma, digestion, headache, and toothache & Magar (Ba) & [23] \\
\hline
\end{tabular}




\begin{tabular}{|c|c|c|c|c|c|}
\hline S.N. & $\begin{array}{l}\text { Scientific name, Nepali name and } \\
\text { Family }\end{array}$ & P.U. & Diseases treated & Ethnic groups & Sources \\
\hline 214. & $\begin{array}{l}\text { Zingiber officinale Roscoe, Aduwa, } \\
\text { Zingiberaceae }\end{array}$ & $\mathrm{Rh}$ & $\begin{array}{l}\text { Throat pain, cough, and high altitude sickness } \\
\text { Cough, vomiting, and infection of caterpillar hairs } \\
\text { Kidney problem, throat pain, gastritis, diabetes, and cough }\end{array}$ & $\begin{array}{l}\text { Raji } \\
\text { Lepcha } \\
\text { Tangbeton }\end{array}$ & $\begin{array}{c}{[25]} \\
{[30,34]} \\
{[32]} \\
{[37]}\end{array}$ \\
\hline 215. & $\begin{array}{l}\text { Ziziphus mauritiana Lam. Bayer, } \\
\text { Rhamnaceae }\end{array}$ & $\mathrm{F}$ & Stomach problem and body cooling & Magar (G) & [14] \\
\hline
\end{tabular}

P.U. $=$ Parts Used, $\mathrm{S}=$ Stem, $\mathrm{B}=$ Bark, $\mathrm{F}=$ Fruit, $\mathrm{L}=$ Leaf, $\mathrm{R}=$ Root, $\mathrm{FI}=$ Flower, $\mathrm{T}=$ Twig, $\mathrm{Rh}=$ Rhizome, $\mathrm{Tu}=$ Tuber, $\mathrm{W}=$ Whole plant, $\mathrm{Bu}=\mathrm{Bulb}$, $\mathrm{Sd}=$ Seed, $\mathrm{Lt}=$ Latex, $\mathrm{Bd}=$ Buds

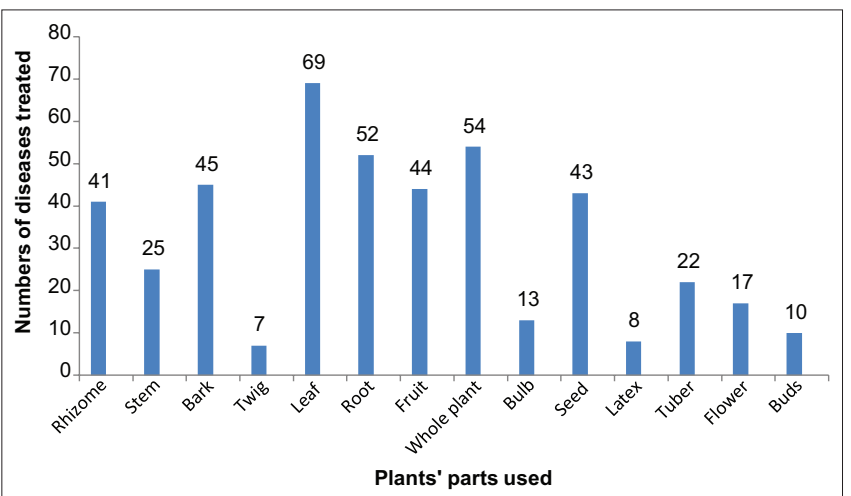

Figure 1: Plants' parts used and numbers of diseases treated

analysis, the leaf is used for the treatment of maximum numbers of diseases (69 diseases) and twig being used for minimum numbers of diseases (7 diseases).

\section{CONCLUSIONS}

Medicinal plants are major sources of crude drugs for healing diseases by various ethnic groups living near the forests of Nepal. A total of 215 Plant species from 93 families is used for the treatment of 139 types of diseases or ailments by 10 ethnic groups residing in 13 hilly districts of Nepal. The whole plant or its parts were used for medicinal purposes and leaf is found to be used for the treatment of maximum numbers of diseases.

Ethnomedicinal knowledge is the foundation for the invention of new medicines. Proper documentation of traditional knowledge on medicinal plants with their identification and cultivation should be done which helps to preserve traditional knowledge as well as medicinal plants. Highly valuable medicinal plants are needed to be grown commercially to reduce pressure on these species in wild habitats. Young generations should be encouraged and trained in traditional medicinal knowledge. Phytochemical and pharmacological researches are needed to be carried out for scientific validation of the medicinal properties of the plant species used by indigenous people.

\section{REFERENCES}

1. Bennett BC. Ethnobotany and Economic Botany: Subjects in search of definitions. Encyclopedia of Life Support Systems; 2002.

2. Budha-Magar S, Bhandari P, Ghimire SK. Ethno-medicinal survey of plants used by Magar (Kham) community, Rolpa district, Western Nepal. Ethnobotany Research and Applications. 2020;19:1-29. doi: http://dx.doi.org/10.32859/era.19.18.1-29

3. Quinlan MB. Ethnomedicine. A companion to medical anthropology; 2011;381-403.

4. Prakash V. Indian medicinal plants; current status. Ethnobotany. 1998;10:112-113.

5. Tomlinson TR, Akerele OEds. Medicinal plants: their role in health and biodiversity. University of Pennsylvania press; 2015.

6. Luitel DR, Rokaya MB, Timsina B, Münzbergová Z. Medicinal plants used by the Tamang community in the Makawanpur district of central Nepal. Journal of Ethnobiology and Ethnomedicine. 2014;10(1):1-11. https://doi.org/10.1186/1746-4269-10-5

7. Rokaya MB, Münzbergová Z, Timsina B. Ethnobotanical study of medicinal plants from the Humla district of western Nepal. Journal of Ethnopharmacology. 2010;130(3):485-504. https://doi.org/10.1016/j. jep.2010.05.036

8. Ghimire SK. Sustainable harvesting and management of medicinal plants in the Nepal Himalaya: current issues, knowledge gaps and research priorities. Medicinal Plants in Nepal: an Anthology of Contemporary Research. 2008; 25-44.

9. Bhattarai KR, Ghimire M. Commercially important medicinal and aromatic plants of Nepal and their distribution pattern and conservation measure along the elevation gradient of the Himalayas. Banko Janakari. 2006;16(1):3-13. https://doi.org/10.3126/banko. v16i1.357

10. Ghimire SK, Mckey D, Aumeeruddy-Thomas Y. Himalayan medicinal plant diversity in an ecologically complex high altitude anthropogenic landscape, Dolpo, Nepal. Environmental Conservation. 2006;128-140. doi: $10.1017 / 50376892906002943$

11. Acharya R, Acharya KP. Ethnobotanical study of medicinal plants used by Tharu community of Parroha VDC, Rupandehi district, Nepal. Scientific world. 2009;7(7):80-84. https://doi.org/10.3126/ sw.v7i7.3832

12. Manandher NP. Ethnobotanical census on herbal medicines of Banke district, Nepal. Contribution to Nepalese studies. 1998;25:57-63.

13. Shrestha PM, Dhillion SS. Medicinal plant diversity and use in the highlands of Dolakha district, Nepal. Journal of ethnopharmacology. 2003;86(1):81-96. https://doi.org/10.1016/S0378-8741(03)00051-5

14. Acharya R. Ethnobotanical study of medicinal plants of Resunga Hill used by Magar community of Badagaun VDC, Gulmi district, Nepal. Scientific World. 2012;10(10):54-65. https://doi.org/10.3126/ sw.v10i10.6863

15. Rahmatullah M, Hasan A, Parvin W, Moniruzzaman M, Khatun A, Khatun Z, Jahan R. Medicinal plants and formulations used by the Soren clan of the Santal tribe in Rajshahi district, Bangladesh for treatment of various ailments. African Journal of Traditional, Complementary and Alternative Medicines. 2012;9(3):350-359. http:// dx.doi.org/10.4314/ajtcam.v9i3.8

16. Kunwar RM, Baral K, Paudel P, Acharya RP, Thapa-Magar KB, Cameron M, Bussmann RW. Land-use and socioeconomic change, medicinal plant selection and biodiversity resilience in Far Western Nepal. PLoS One. 2016;11(12):e0167812. https://doi.org/10.1371/ journal.pone.0167812

17. Singh AG, Kumar A, Tewari DD. An ethnobotanical survey of medicinal plants used in Terai forest of western Nepal. Journal of ethnobiology and ethnomedicine. 2012;8(1):19. https://doi.org/10.1186/1746-42698-19

18. CBS. National population and housing census 2011, National Report. Kathmandu, National Planning Commission2012. 2012.

19. Rana SK, Oli PS, Rana HK. Traditional botanical knowledge (TBK) on 
the use of medicinal plants in Sikles area, Nepal. Asian Journal of Plant Science and Research. 2015;5(11):8-15. www. pelagiaresearchlibrary. com

20. Thapa S. Medico-ethnobotany of Magar community in Salija VDC of Parbat district, central Nepal. Our nature. 2012;10(1):176-190. https:// doi.org/10.3126/on.v10i1.7780

21. Singh AG, Kumar A, Tewari DD, Bharati KA. New ethnomedicinal claims from Magar community of Palpa district, Nepal. 2018. http:// nopr.niscair.res.in/handle/123456789/44583

22. Uprety $Y$, Poudel RC, Asselin H, Boon E. Plant biodiversity and ethnobotany inside the projected impact area of the Upper Seti Hydropower Project, Western Nepal. Environment, Development and Sustainability. 2011;13(3):463-492. https://doi.org/10.1007/ s10668-010-9271-7

23. Sapkota PP. Ethno-ecological Observation of Magar of Bukini, Baglung, Western, Nepal. Dhaulagiri Journal of Sociology and Anthropology. 2008:2:227-252. https://doi.org/10.3126/dsaj.v2i0.1366

24. Balami NP. Ethnomedicinal uses of plants among the Newar community of Pharping village of Kathmandu district, Nepal. Tribhuvan University Journal. 2004;24(1):13-19. https://doi. org/10.3126/tuj.v24i1.251

25. Paudel M. Medical Ethnobiology and Indigenous Knowledge System Found In Raji Group of Nepal (Doctoral dissertation, Central Department of Zoology Institute of Science and Technology Tribhuvan University Kirtipur, Kathmandu, Nepal); 2015.

26. Shrestha P. Contribution to the ethnobotany of the Tamangs of Kathmandu valley. Contributions to Nepalese Studies. 1988; 15(2):247-266.

27. Tamang G. An ethnobiological study of the Tamang people. Our nature. 2003;1(1):37-41. https://doi.org/10.3126/on.v1i1.303

28. Bhattarai KR. Ethnobotanical study of plants used by Thami community in Ilam District, eastern Nepal. Our Nature. 2018;16(1):5567. https://doi.org/10.3126/on.v16i1.22123

29. Subba B, Srivastav C, Kandel RC. Scientific validation of medicinal plants used by Yakkha community of Chanuwa VDC, Dhankuta, Nepal. Springerplus. 2016;5(1):155. https://doi.org/10.1186/s40064016-1821-5

30. Bhattarai KR. Ethnomedicinal practices of the Lepcha community in Ilam, east Nepal. Journal of Plant Resources. 2017;15(1):31-44

31. Rai R, Singh NB. Medico-ethnobiology in Rai community: a case study from Baikunthe Village development committee, Bhojpur, eastern Nepal. Journal of Institute of Science and Technology. 2015;20(1):127132. https://doi.org/10.3126/jist.v20i1.13935

32. Paudyal R, Singh NB. Ethno-medicinal uses of animals and plants among the migratory tangbetons of Pokhara, Nepal. Journal of Institute of Science and Technology. 2014;19(1):145-149. https:// doi.org/10.3126/jist.v19i1.13840

33. Singh AG, Gautam LP, Tewari DD. Folk uses of some medicinal plants of dobhan VDC of Palpa district, Western Nepal. Journal of Phytology. 2011.

34. Tamang $P$, Singh NB. Medical ethnobiology and indigenous knowledge system of the Lapcha of Fikkal VDC of llam, Nepal. Journal of Institute of Science and Technology. 2014;19(2):45-52. https://doi. org/10.3126/jist.v19i2.13851

35. Thapa LB. Indigenous knowledge on common medicinal plants among Raji community of Surkhet District, Mid-Western Nepal. Nepalese Journal of Biosciences. 2012;2:88-92. https://doi. org/10.3126/njbs.v2i0.7494 